\title{
Grenzenlose Karrieren? - Hochqualifiziertes Personal und Top-Führungskräfte in Ökonomie und Medizin
}

\author{
Markus Pohlmann • Stefan Bär
}

Zusammenfassung: Ziel des Beitrags ist es, die These ,grenzenloser Mobilität“"von Hochqualifizierten zu prüfen, die davon ausgeht, dass es im Zuge der Globalisierung zu einer internationalen Verteilung und zu transnationalen Rekrutierungsstrukturen des hochqualifizierten Personals komme. Wir betrachten auf der Basis eigener empirischer Untersuchungen und der Sekundärauswertung weiterer Studien die Wanderungsdynamik Hochqualifizierter in zwei institutionellen Feldern: Ökonomie und Medizin näher und kommen zu dem Ergebnis, dass sich diese „starke“ Globalisierungsthese nicht halten lässt. Wir sprechen daher von „Internationalisierung light“ und verorten die Gründe dafür auf der Ebene organisationaler Karrieremechanismen.

Schlüsselwörter: Globalisierung · Internationale Mobilität · Hochqualifiziertes Personal · Führungskräfte $\cdot$ Karrieremechanismen

\section{Bondless Careers?}

Abstract: The aim of the article is to prove the hypothesis of "bondless mobility" of the highly qualified. It says, that there evolved international distribution and trans-national structures of recruiting of the highly qualified personnel as part of the globalisation. We take our focus on managers and physicians, and based on our own and secondary analyses of other studies we find that this "strong" hypotheses of globalisation does not appear tenable. We prefer the term "Internationalisierung light" and locate the reasons for that in organisations' career-mechanisms.

Keywords: Globalisation · International Mobility · Highly Qualified Personnel · Executives · Career-mechanisms

Prof. Dr. Markus Pohlmann $(\bowtie)$ Ruprecht-Karls-Universität Heidelberg Institut für Soziologie Bergheimer Straße 58 69115 Heidelberg, Deutschland E-Mail: markus.pohlmann@soziologie. uni-heidelberg.de

\author{
Dipl.-Soz. Stefan Bär $(\bowtie)$ \\ Ruprecht-Karls-Universität Heidelberg \\ Institut für Soziologie \\ Bergheimer Straße 58 \\ 69115 Heidelberg, Deutschland \\ E-mail: stefan.baer@soziologie. \\ uni-heidelberg.de
}

\section{Erstmals erschienen in:}

Österreichische Zeitschrift für Soziologie. Volume 34, Nr. 4 / Dezember 2009, S. 13-40. 
Für viele Hochqualifizierte ${ }^{1}$ bedeutet Globalisierung nicht nur zunehmende Unsicherheit in Bezug auf Konkurrenz und richtige Karrierewege (vgl. dazu auch die neuere Studie von Kotthoff/Wagner 2008), sondern auch, dass sich für die eigene internationale und soziale Mobilität neue Chancen eröffnen. Während gegenüber der aus der Not geborenen Arbeitsmigration nicht selten restriktive Migrationspolitiken der Nationalstaaten greifen, sind der Migration von Hochqualifizierten Tür und Tor geöffnet (vgl. dazu u. a. Dreher 2003: 18; Chalamwong 2005: 488). Für sie besteht die Welt nicht - wie für die Mehrheit der Migranten - aus territorial abgegrenzten Staatsräumen mit „Non-Entree“Politiken, sondern als hochqualifizierte Minderheit können sie sich einen denationalisierten Raum mit vielen „Entree“-Angeboten frei erschließen. ${ }^{2}$ Ein verschärfter internationaler Wettbewerb des Personals um begehrte Jobs und ein Kampf von Unternehmen und Nationen um die weltweit „,besten Köpfe“ seien die Folge. Insbesondere Professionals und Manager erscheinen als Vorreiter dieser Entwicklung - die Prototypen in einem Prozess der „Enträumlichung, in dem Geld, Waren und Personen einander rast- und ruhelos um die Welt jagen“ (Appadurai 1998: 15). So breite sich durch die Globalisierung eine Art „Jetset“ des hochqualifizierten Personals weiter aus, eine als ebenso international erfahrene wie weltoffen illuminierte neue „Weltklasse“ (vgl. dazu auch Pohlmann 2003; 2008).

Wer nicht längere Zeit im Ausland war, so scheint es, der bleibt zurück. Mobilitätsbereitschaft erscheint heute als Voraussetzung für steile Karrieren, und je größer diese ist, so das ungeschriebene Gesetz, desto steiler kann die Karriere werden. Insbesondere die deutschen Studierenden machen es vor. Noch nie haben so viele von ihnen im Ausland studiert. Im europäischen Vergleich belegen sie Spitzenplätze, weltweit steht Deutschland als Entsendeland nach China, Indien, Südkorea und Japan an fünfter Stelle (BMBF 2005: 9 f.). Die Unsicherheit in Bezug auf die eigenen Karrieren ist groß, und ein Auslandsjahr oder mehr erscheint hier als sicheres Karrieresprungbrett, Sprachkenntnisse inklusive. Aber auch die Anzahl der ins Ausland entsandten Firmenmitarbeiter hat stark zugenommen. Sie bilden eine neue Form der Migration, die offensichtlich anderen Regeln folgt als die Auswanderungen (vgl. dazu Kolb 2006 u. a.). Die Rede vom intensivierten globalen Wettbewerb um Professionals und Führungskräfte legt es nahe: In transnationalen Organisationen sind auch die Karrieren transnational.

Wir begeben uns in den folgenden Ausführungen daher auf die Spur der Migration von Professionals und Führungskräften und fragen nach, wie es um die gestiegene Mobilitätsbereitschaft, die Karrieremuster und deren Internationalisierung in Deutschland tatsächlich bestellt ist. Dabei beziehen wir uns auf Untersuchungsergebnisse aus zwei Forschungsprojekten (siehe Anhang I) sowie die Ergebnisse verschiedener anderer geographischer und soziologischer Studien. Unsere Hypothese hierbei ist, dass diese Internationalisierung der Hochqualifizierten- und Managerkarrieren vor der Internationalisierung der Unternehmen zurückbleibt, weil nicht die viel zitierten globalen Arbeitsmärkte, sondern die organisationalen Karrieresysteme der Unternehmen die Karriereverläufe prägen. Nicht die „starke“ Globalisierungsthese lässt sich aufrechterhalten, so unsere Annahme, sondern nur ihre „schwächere“ Variante: dass die internationale Mobilität des hochqualifizierten Personals zwar im Zuge der Globalisierung der Wirtschaft zugenommen hat, aber den Regeln des national geprägten Karrieresystems weiterhin folgt. Wir stellen dazu zunächst die gängigen Annahmen zur Internationali- 
sierung des hochqualifizierten Personals vor (1.) und prüfen anschließend die im Globalisierungsdiskurs formulierte These, dass die Globalisierung in der Tendenz zu einer „grenzenlosen Mobilität“ der Hochqualifizierten führe. Wir nehmen dies wörtlich und nehmen hierzu die nationalen Grenzen der BRD ins Visier und fragen anhand verschiedener geographischer Studien, welche Bedeutung diese für die Strukturierung der Arbeitsmigration tatsächlich hat (2.). Diese in der Tendenz ,grenzenlose Mobilität“ sorge, so die Fortsetzung der These, für einen „war of talents“, einen Kampf um die besten Köpfe. Dieser hat zwei Seiten, die in der Diskussion sind: zum einen die Gefahr einer Abwanderung (,brain drain“) der „besten Köpfe“ in Länder mit besseren Konditionen (wie z. B. in die USA) und zum anderen die Möglichkeiten ihrer Anwerbung durch vereinfachte Migrationsbedingungen und einfachen Zugang zu den Staatsbürgerechten. Wir fassen dabei den „brain-drain“ als „freiwillige internationale Einzelmigration hochqualifizierter Arbeitskräfte" und unterscheiden nach der Richtung der Wanderungsbewegung die Abwanderung (,brain drain“) und die Einwanderung (,brain gain“), wobei wir die von vorne herein zeitlich befristete Mobilität als „brain circulation" bezeichnen, auch wenn damit Entsendungen von mehreren Jahren inbegriffen sind (vgl. im Unterschied dazu Galinski 1986). Von einem „brain drain“ kann u. E. in substanzieller Weise nur gesprochen werden, wenn die Karrieren auch im Ausland fortgesetzt werden und der Auslandsaufenthalt nicht nur als „Durchlauferhitzer“ für die inländische Karriere dient. Vor diesem Hintergrund interessiert uns in diesem Artikel nicht die politisch-institutionelle oder rechtliche Seite - also die nationalen Strategien, mit denen dieser ,war of talents“ ausgefochten wird -, sondern vielmehr, ob er zwischen den entwickelten OECD-Ländern überhaupt in einem Sinne stattfindet, dass er die Abwanderungs- und Einwanderungsdynamik der Hochqualifizierten prägt. Wir konzentrieren uns dabei in diesem Artikel auf Deutschland, haben aber auch zu Ostasien, Europa und den USA entsprechende Vergleichsdaten untersucht und analysiert (Pohlmann 2009). In Deutschland dreht sich diese Diskussion vor allem um drei hochqualifizierte Beschäftigtengruppen: Wissenschaftler allgemein sowie insbesondere Ingenieure, Medizinisches Personal und Führungskräfte in der Wirtschaft. Da zu Wissenschaftlern und Ingenieuren bereits einige Studien vorliegen, konzentrieren wir uns hier zunächst auf das hochqualifizierte, medizinische Personal und versuchen mittels sekundäranalytischen Auswertungen deren Ab- und Einwanderungsdynamik in Deutschland zu analysieren (3.). Danach wenden wir uns der These der ,transnationalen Migration“"von Professionals und Führungskräften in der Wirtschaft zu und nutzen dazu Sekundäranalysen $^{3}$ und Interviewmaterial einer von der DFG geförderten Studie zur ökonomischen Elite ${ }^{4}$ in Deutschland. Wir verstehen auf der Basis des Positionsansatzes unter „transnationalen Karrieren“ Positionseinstiege oder -aufstiege von (hier: hochqualifiziertem) Personal, das seine Karriere außerhalb des Landes, in dem es aufgewachsen ist, kontinuierlich (d. h. ohne vorherige zeitliche Befristung) fortsetzt. Im Falle der „transnationalen Manager“ bekleiden diese Managementpositionen in Unternehmen, die nicht zu einer Mutterfirma des Heimatlandes gehören. Auch hier wird gefragt, ob sich die so verstandene transnationale Migration tatsächlich so entwickelt, wie es die Globalisierungsliteratur propagiert (4.). In unserem Fazit versuchen wir, neben den üblichen ökonomischen Erklärungsfaktoren auch erste soziologische Erklärungen für diese Befunde anzudeuten (5.). 


\section{Globalisierung und Internationalisierung von Hochqualifizierten}

Dem Thema der Entstehung eines globalen Managements von Wirtschaftsunternehmen und auch Krankenhäusern sind viele Artikel und Proklamationen gewidmet, aber nur wenige Autoren haben die Internationalisierung des hochqualifizierten Personals in Medizin und Ökonomie systematisch untersucht. Nichtsdestotrotz hat die Politik eine klare Diagnose gefunden: So berichtete z. B. die Unabhängige Kommission „Zuwanderung“ des Bundesministeriums des Innern in Deutschland, dass ein weltweiter „Wettbewerb um die besten Köpfe“ entstanden sei, der durch die gestiegene Mobilitätsbereitschaft dieser Personen verschärft werde (BMI, Unabhängige Kommission „Zuwanderung“ 2001). Dieser Aussage konnte sich die deutsche Bundesregierung anschließen (Schäuble 2007) ${ }^{5}$. Sie kann angesichts der voranschreitenden Internationalisierung der Unternehmen eine hohe Plausibilität für sich beanspruchen, und auch im „Mainstream“ der Globalisierungsliteratur ${ }^{6}$ ist klar, dass die Internationalisierung von Professionals und Führungskräften jener der Unternehmen und auch der Krankenhäuser folgt. Auch bei den Diskussionen um den Arztberuf in Deutschland wird in den letzten Jahren immer wieder hervorgehoben, dass es um die Arbeitsbedingungen und Verdienstmöglichkeiten im internationalen Vergleich schlecht bestellt sei und dass deswegen immer mehr deutsche Ärzte ins Ausland abwanderten (vgl. bspw. Kopetsch 2008; Richter-Kuhlmann 2008). Die Attraktivität des Arztberufes schwinde, und es werde daher vor dem Hintergrund eines zurückgehenden Nachwuchses schon bald zu einem Ärztemangel und zu Versorgungsengpässen kommen. ${ }^{7}$ Auch hier wird die Entstehung eines transnationalen sozialen Raumes angenommen, in welchem die „neuen“ hochqualifizierten Arbeitsmigranten der Medizin jenseits nationaler Grenzen und Bindungen sich bewegen, arbeiten und leben.

Insgesamt führe die radikale Globalisierung der Ökonomie durch die Zunahme von Anzahl und Bedeutung multi- und transnationaler Unternehmen zu einer Internationalisierung des Personals und zur Entstehung einer transnationalen Elite (vgl. dazu Moss Kanter 1999; Sklair 2001; zu den transnationalen Unternehmen: Bartlett/Ghoshal 2001; Pries 2006). Dahinter stehen eine gestiegene Mobilitätsbereitschaft und zunehmend internationale Rekrutierungsmuster der Professionals und Führungskräfte. Zwar wird diese Entwicklung kritisch kommentiert, aber zugleich die Entstehung einer „neuen Weltklasse“ des Managements annonciert (Beck 1997: 17).

In der Migrationssoziologie wird den Hochqualifizierten geringere Aufmerksamkeit geschenkt. ${ }^{8}$ Arbeitsmigration wird häufig unter der Frage der Integration in die Aufnahmegesellschaft thematisiert und das Problem der geringer Qualifizierten oder ethnisch Diskriminierten bei der Analyse der globalen Wanderungsprozesse besonders betont (vgl. dazu Pries 1998: 71; Pries 2003; 2005b; Kolb 2006). Zwar hat im letzten Jahrzehnt die Thematisierung von transnationaler Migration zugenommen, sie konzentriert sich aber eher auf gering qualifizierte Migration zwischen bestimmten Weltregionen. ${ }^{9}$ Wenn Manager und Hochqualifizierte thematisiert werden, dann wird auch hier davon ausgegangen, dass ein neuer internationaler Arbeitsmarkt in Expansion begriffen sei (vgl. dazu Pries 1998, Castles/Miller 1993, Rodriguez-Pose 2003). Rodriguez-Pose (2003: 98-100) diagnostiziert für die Europäische Union einen Boom der Migration von Hochqualifizierten, der durch den Wegfall von bürokratischen Schranken und die Etablierung 
des Binnenmarktes ,getriggert“ sei, ohne diesen allerdings mit Daten zu belegen. Dabei werden die Bewegungen von Arbeitskräften zwischen Unternehmen zwar als neue Form der Migration registriert, aber selten in ihrer Bedeutung abgeschätzt (so auch Kolb 2006: 162; Salt 1987; 1988; 1989: 451 f.). Klar erscheint in der Diskussion, dass die steigenden, aber absolut betrachtet eher geringen Zahlen, nichts über die ökonomische Bedeutung dieser Migrationsform aussagen (vgl. ebd.; Hillmann/Rudolph 1997). Diese jedoch zu bestimmen, fällt den neueren Migrationsansätzen schwer. Bezogen auf das Management bleibt es bei ersten Plausibilisierungen der These seiner Transnationalität. „Transnationalität" wird hier sehr weit dadurch definiert, dass sich Migranten soziale Felder zwischen Ankunfts- und Herkunftsregion erschließen und dadurch transnationale „communities“ oder soziale Räume entstehen, die für die alltägliche Lebenspraxis wichtig sind und über den Sozialzusammenhang des Nationalstaates hinausweisen (vgl. dazu Glick Schiller 1992; Glick Schiller et al. 1995; Pries 1998). Auf die Frage aber, inwieweit Mechanismen oder Regeln der Auswahl und Beförderung dieses Personals neben den nationalen und unternehmensspezifischen Karrieresystemen entstanden sind, welche die national geprägten Karrieresysteme durch globale Zugangs-, Zuweisungs- und Mobilitätsmöglichkeiten transzendieren, durchbrechen oder ergänzen, stehen hinreichend empirisch fundierte Antworten noch aus. Auch die betriebswirtschaftliche Literatur zum ,transnationalen Management“ definiert eher die richtige transnationale Strategie der Unternehmensführung, als die Karrieren der Führungskräfte selbst einer systematischen Untersuchung zu unterziehen (vgl. Bartlett/Ghoshal 2001u.v. a.).

\section{Grenzenlose Mobilität? Zur Bedeutung von nationalen Grenzen in Deutschland}

Die Vorstellung einer forcierten Internationalisierung von Führungskräften und Professionals wird empirisch getragen durch eine stärker artikulierte Bereitschaft zur Mobilität sowie eine erhöhte tatsächliche räumliche Mobilität, insbesondere der jüngeren und höher qualifizierten Arbeitskräfte. ${ }^{10}$ Wir wollen im Folgenden zeigen, dass dieses Bild von der ,grenzenlosen“ Mobilität des Humankapitals und der damit verbundenen Entstehung internationaler Arbeitsmärkte sowie der Erosion des Nationalstaates vom Mainstream der Globalisierungsliteratur überzeichnet ist. Dazu konzentrieren wir uns in diesem Kapitel zunächst auf die Bedeutung nationaler Grenzen für die räumliche Mobilität, um die Vorstellung der „Grenzenlosigkeit“ der Mobilität des Humankapitals zu überprüfen. Wir beziehen uns dabei - neben den Daten des SOEP - auf eine Reihe von geographischen Studien, die diese Wanderungsbewegungen über die Grenzen genauer in den Blick genommen haben.

Betrachten wir im ersten Schritt der Argumentation die Bedeutung der nationalen Grenzen für Grenzpendler und Arbeitsmigranten aus Deutschland und den Nachbarländern insgesamt, so kommen die geographischen Studien zu sehr klaren Befunden. Sie legen für Deutschland eher den Schluss nahe, dass deren „politisches Aufweichen“ im Zuge der Globalisierung und des ,war of talents“ an ihrem Einfluss auf die grenzregionalen Wanderungsbewegungen wenig geändert hat. Dies wird ebenfalls durch OECDDaten bestätigt. 
Wie scharf für alle Bewohner von Grenzregionen - ob gering oder hoch qualifiziert nationale Grenzen greifen, zeigen diese Untersuchungen exemplarisch in Bezug auf die Mobilität zwischen Deutschland, Dänemark, den Niederlanden, Tschechien und der Großregion im Grenzgebiet Belgien - Deutschland - Luxemburg - Frankreich. So kamen die im Jahre 1990/91, 1995 und 1998 durchgeführten Untersuchungen der Grenzregion Flensburg-Sønderjylland zu dem Schluss, dass das Pendeln über die Grenze einen Umfang hatte, der zehnmal geringer war, als zu erwarten gewesen wäre, wenn es keine Grenze gäbe (vgl. Haller et al. 2000: 6). Die Zahl der Grenzpendler lag noch im letzten Jahr der Erhebung, 1998, anteilsmäßig bei unter einem Prozent der Beschäftigten in der Region (ebd.). Auch bei der Arbeitsmigration in Mittel-Ost-Europa entsprechen die Pendlerströme nach diesen Analysen nicht dem häufig propagierten Bild der Medien. Obwohl im deutsch-tschechischen Grenzgebiet die Migrationsbereitschaft, Umfragen zufolge, relativ hoch ist ( 30 bis $40 \%$ der befragten Tschechen gelten als potentielle Migranten), ist die Arbeitsmigration insgesamt eher gering. Lediglich 3\% realisieren dieses Vorhaben, welches sich zudem in der Mehrzahl als „Pendeln“ und Saisonarbeit darstellt (Jerábek, Dokoupil 2003). Für polnische Migranten stellt Deutschland zwar ein Hauptzielland dar, der Anstieg von 131.000 auf 307.000 polnische Arbeitskräfte in den Jahren von 1992 bis 2004 (OECD Development Center 2007: 109) beruht u. a. auf der Tatsache, dass die Europäische Union schon vor dem Beitritt Polens die deutsch-polnische Grenzregion mit Projekten gefördert hatte. ${ }^{11}$ Der größte Teil dieser Arbeitsmigration ist jedoch ebenfalls Saisonarbeit und wird als „,circular migration“ bezeichnet (ebd.: 44).

Auch in der „Euregio Bodensee“ beträgt der Anteil der Grenzpendler nur 1,8\% der Beschäftigten (gegenüber durchschnittlich 1,4\% im europäischen Vergleich). 2001 pendelten täglich 35.000 Personen über die Grenzen zwischen Deutschland, Lichtenstein, Österreich und der Schweiz zu ihren Arbeitsstellen (Schulz 2005: 6). Die Beobachtungen der Pendlerströme weisen im Vergleich zu 1990 einen leichten Rückgang um 5\% aus (ebd.: 8). Deutschland liegt als Zielland für diese regionale Gruppe der Grenzgänger mit etwa 1.800 Personen, davon stammt der größte Teil aus Österreich, am unteren Rand der Verteilung. Die größten Ströme von Pendlern und Arbeitsmigranten verlaufen zwischen der Schweiz (Sankt Gallen), Österreich (Vorarlberg) und dem Fürstentum Liechtenstein. Lediglich 0,3\% der Beschäftigten in der deutschen Grenzregion sind Einpendler (ebd.: 20,23). Dagegen pendeln umgekehrt fast $5 \%$ der Erwerbstätigen auf deutscher Seite bspw. aus dem Landkreis Konstanz in die Schweiz (ebd.: 23). Bis auf den Kanton Zürich haben sich in der Schweiz die Einpendlerzahlen im Vergleich zu 1990 leicht verringert, wofür neben dem Strukturwandel mit Verschiebungen der Pendlerzahlen vom sekundären in den tertiären Sektor eine Erleichterung der Verlagerung des Wohnsitzes durch bilaterale Verträge als Ursache angenommen wird (ebd.: 25). Noch für die als pendelintensiv benannte Großregion Saarland - Rheinland-Pfalz - Lothringen - Luxemburg - Wallonien zeigt sich, dass es lediglich 4\% der Arbeitnehmer der Grenzregionen Belgiens, Deutschlands, Luxemburgs und Frankreichs (Interregionale Arbeitsmarktbeobachtungsstelle 2005: $18 \mathrm{ff}$.) sind, die dafür verantwortlich zeichnen.

Erweitert man die Betrachtungsebene und fragt angesichts dieser Ergebnisse, wie viele der EU-Bürger insgesamt im Ausland leben - und dabei lassen wir zunächst die Einbürgerungen außer Acht -, so zeigt sich, dass es nur 1,5\% aller EU-Bürger sind. Dabei 
macht die ehemalige Gastarbeitergeneration neben den Familienzusammenführungen der letzten Jahrzehnte den größten Anteil aus. Weniger als 0,2\% der EU-Bürger sind, Analysen von Rother (2005: 41) zufolge, im Jahr 2000 in ein anderes EU-Land gezogen. Die Umzüge von Bundesbürgern innerhalb der Bundesländer betragen im Vergleich dazu 1,4\% (ebd.).

Folgt man den Ergebnissen der verschiedenen Studien ergibt sich für Deutschland ein Bild, das die Bedeutung von nationalen Grenzen für Pendler und Arbeitsmigranten eher unterstreicht, als sich der Vorstellung der „Grenzenlosigkeit“ einfach zu fügen.

Im zweiten Schritt wollen wir nun schauen, wie sich unterschiedliche Qualifikationsniveaus auf die Bedeutung der grenzüberschreitenden Mobilität auswirken. Dabei zeigt sich, dass Nicht-Akademiker in wesentlich geringerem Ausmaß mobil sind als Akademiker. Den verfügbaren repräsentativen Panel-Untersuchungen zufolge ist die Mobilität der Nicht-Akademiker in Deutschland - aber zum Beispiel auch in den USA ${ }^{12}$ - selbst dann außerordentlich gering, wenn Arbeitslosigkeit vorliegt. Bemerkenswert sei die Tatsache, so Büchel, dass (jeweils auf das Vorjahr bezogen, d. V.) Arbeitslose nicht häufiger aus beruflichen Gründen umziehen als Nichtarbeitslose (Büchel et al. 2002: 219). Nach den repräsentativen Daten des $\mathrm{SOEP}^{13}$ endet die vermeintlich ,grenzenlose Mobilität" für Nicht-Akademiker in Deutschland (und auch in den USA) im Regelfall an den Grenzen des eigenen Landkreises oder der eigenen Gemeinde.

Auch eine Betrachtung der - insgesamt deutlich mobileren - Hochqualifizierten ändert aber an dieser grundlegenden Bedeutung nationalstaatlicher Grenzen für die Migrationsdynamik nichts. Für die Akademiker lässt sich nach den neuesten Daten des SOEP (Datensatz 2007/2008) sagen, dass auch diese nur in sehr geringem Maße ins Ausland pendeln. Von den 20.886 Befragten des SOEP-Datensatzes besitzen zwar 4.335 einen Hochschulabschluss ${ }^{14}$, aber nur 52 Personen haben ihren Arbeitsplatz im Ausland, zu welchem sie in der Mehrzahl täglich bzw. wöchentlich pendeln. Das entspricht bei den Hochschulabsolventen 0,43\%, bzw. 0,25\% in der Gesamtstichprobe. Differenziert man die kleine Gruppe der Grenzpendler ins Ausland nach der beruflichen Stellung, dann zeigt sich, dass neben Arbeitern, Facharbeitern, einigen Selbständigen und Beamten nur marginal wenige Angestellte in Führungspositionen und Leitungspositionen darunter vertreten sind (insgesamt 8 ).

Eine zum Stichjahr 1997 von Geographen durchgeführte Untersuchung zur Mobilität von Personen mit hohen schulischen Abschlüssen kommt für die Grenzregionen zu Dänemark und den Niederlanden zu ähnlich geringen Pendler- und Abwanderungsbewegungen. Zirka 40\% der 2.203 deutschen und niederländischen Befragten, die 15 Jahre zuvor Abitur oder die holländischen Abschlussexamina gemacht hatten (Jahrgänge von 1982/1983), waren in der Herkunftsregion verblieben und 55\% innerhalb der Grenzen des Nationalstaates mobil. Nur jeweils ein Prozent war von Deutschland in die Niederlande oder umgekehrt abgewandert. Und noch weniger, jeweils nur $0,5 \%$, pendelten in die Grenzregion des jeweiligen Nachbarlandes (Janssen 2001: 2). Damit erweist sich insgesamt - trotz der gewachsenen Freizügigkeit in der EU - die grenzüberschreitende Mobilität auch der Hochqualifizierten als relativ gering.

Wenn man fragt, woraus diese nationalen Grenzen berufsbedingter Mobilität gemacht sind, erhält man unterschiedliche Antworten. Während die Nichtmobilen selbst häufig weiche Faktoren wie fehlende Sprach- und Kulturkenntnisse und fehlende Informatio- 
nen über den Arbeitsmarkt für eine geringere Mobilität verantwortlich machen, verweisen Unternehmer und Arbeitgeber eher auf Faktoren, die auf unterschiedliche institutionelle Settings zurückgehen (Haller et al. 2000: 13 f.; Janssen 2001: 4 f.). Insbesondere die Unterschiede in den Sozialversicherungs- und Steuersystemen sowie die fehlende Vergleichbarkeit in den Bildungsabschlüssen spielen dabei eine entscheidende Rolle (Haller et al. 2000: 14). Dies wird von der Literatur weitgehend bestätigt (vgl. Janssen 2001: 4). Selbst die Kommission der Europäischen Gemeinschaften stellt (2005) in ihrem Vorschlag für eine Richtlinie des Europäischen Parlamentes und des Rates fest, dass beispielsweise die unterschiedlichen Regelungen der Zusatzsysteme zur Altersversorgung Mobilitätshindernisse darstellen. Auch Fassmann und Meusburger (1997) sehen die europäischen Migrationsmuster als „, . . . ] durch kulturelle, politische und historische Verbindungen zwischen bestimmten Herkunfts- und Zuwanderungsgesellschaften überformt [. . .]" (ebd.: 188). Institutionelle Settings, aber auch, wie wir im Folgenden zeigen wollen, organisationale Karrieresysteme schaffen hier offenbar Pfadabhängigkeiten, die der grenzenlosen Mobilität von Arbeitskräften in Deutschland und seinen Nachbarländern entgegenstehen.

\section{Die Migration von Hochqualifizierten und Eliten in der Medizin}

Die Tatsache, dass die Medizin als Wissenschaft sehr international ist, hat in Deutschland zu der Vermutung geführt, dass wir aufgrund besserer Bezahlung und Arbeitsbedingungen im Ausland insbesondere bei dieser Profession eine Abwanderungsdynamik zu vergegenwärtigen hätten. In Presse und Politik jedenfalls scheint dieser „,brain drain" von Medizinern eine ausgemachte Sache. Diese Vorstellung soll im Folgenden geprüft werden. Welche Rolle spielen „brain drain“- und „brain gain“-Dynamiken tatsächlich für die medizinische Profession in Deutschland? Um diese Frage beantworten zu können, beziehen wir uns zum einen sekundäranalytisch auf die Statistiken der Bundes- und Landesärztekammern und ergänzen dieses Material zum anderen durch eigene Untersuchungen zu Chefärzten und ärztlichen Direktoren großer Kliniken in Deutschland.

\subsection{Verliert Deutschland seine besten Ärzte? Zur Frage des „brain drain“}

In der Frage des „brain drain“ der Mediziner, also der Fortsetzung ihrer Karriere im Ausland, zeigen die Daten der Bundesärztekammer zunächst, daß im Jahr 2007 insgesamt 2.439 Ärzte aus Deutschland ins Ausland abgewandert sind. 77\% dieser Ärzte hatten die deutsche Staatsangehörigkeit (vgl. BÄK 2007), 23\% sind Rückkehrer in ihr Heimatland. Wir sprechen also von knapp 1.900 ,ausgewanderten“ deutschen Ärzten im Jahr 2007. Dabei ist die Gesamtzahl gegenüber 2006 um 5,3\% gesunken. Tatsächlich hat im Jahr 2007 die Migration deutscher Ärzte wohl eher abgenommen. Es bleibt allerdings unklar, in welchem Umfang Rückwanderungen die Dynamik der Ärztemigration bestimmen. Ob der Anteil deutscher Auswanderer konstant bleibt, zu- oder abnimmt, lässt sich nicht einfach feststellen. ${ }^{15}$ Auch wenn man bei allen Unwägbarkeiten der statistischen Erfas- 
sung in den Auswanderungsländern von einigen Unschärfen ausgehen muss ${ }^{16}$, so zeigt die geschätzte Gesamtzahl der im Ausland tätigen 15.916 (vgl. Kopetsch 2007) bzw. 19.054 (vgl. Kopetsch 2008) deutschen Ärzte im Vergleich zur Zahl ausländischer Ärzte in Deutschland, dass sich Ein- und Auswanderungen die Waage halten bzw. dass Deutschland zumindest in der Vergangenheit wohl eher ein Nettoimporteur von Ärzten gewesen ist.

Auch ist bei der Interpretation der Wünsche von Jungmedizinern nach einer Übersiedelung ins Ausland Vorsicht geboten. Einen Teil der Facharztausbildung oder einen befristeten Forschungsaufenthalt im Ausland zu verbringen, können sich Richter-Kuhlmann (2007) zufolge 88\% (Facharztausbildung) bzw. 42\% (Forschung) der Befragten vorstellen. Genau dieses Bild ergibt sich, wenn man auf die Realisierung von Weiterbildungschancen im Ausland blickt. Es gibt eine Vielzahl von Gastärzten aus Deutschland, aus Österreich oder der Schweiz (2007), die ihre Karriere nicht im Ausland fortsetzen, sondern den Auslandsaufenthalt als Karrieresprungbrett für das Inland nutzen. Viel mehr ist auch die Weiterbildung in Deutschland für viele ausländische Absolventen nicht (vgl. Gieseke 2008).

Die Zielländer deutscher ,auswandernder" Ärzte sind Großbritannien, USA, Schweiz $^{17}$, Österreich und Schweden. 2007 wanderten in die Schweiz 684, nach Österreich 269, nach den USA 195 und nach Großbritannien ${ }^{18} 101$ deutsche Ärzte aus (vgl. BÄK 2007).

Tabelle 1: Anzahl deutscher Ärzte im Ausland, aufgelistet nach Ländern

\begin{tabular}{lrc}
\hline Land & Anzahl & Jahr \\
\hline Großbritannien & 4.129 & 2007 \\
USA & 2.694 & 2001 \\
Schweiz & 2.565 & 2007 \\
Österreich & 1.457 & 2007 \\
Schweden & 1.116 & 2005 \\
Frankreich & 975 & 2006 \\
Norwegen & 650 & 2004 \\
Niederlande & 584 & 2007 \\
Italien & 538 & 2001 \\
Belgien & 338 & 2007 \\
Spanien & 259 & 2001 \\
Luxemburg & 116 & 2001 \\
Portugal & 106 & 2004 \\
Irland & 105 & 2004 \\
Dänemark & 87 & 2004 \\
Finnland & 65 & 2006 \\
\hline Summe & 15.916 & \\
\hline
\end{tabular}

Quelle: Kopetsch 2007 
Die Auswanderung deutscher Ärzte einzig und allein nach der absoluten Zahl zu beurteilen, verstellt allerdings den Blick auf den tatsächlichen Trend. Eine Bezugnahme auf die Entwicklung der Gesamtbeschäftigungssituation von Ärzten ist hier wichtig. Denn diese hat sich deutlich verändert. Dieser tatsächliche „Auswanderungs“-Trend ist, anteilig gemessen an der Zahl der berufstätigen Ärzte (vgl. Tabelle 2), bis zum Jahr 2004 gestiegen und liegt seither mit Schwankungen unter diesem Höchstwert, welcher sich allerdings unterhalb einer Marke von $1 \%$ befindet. Von einer Auswanderungstendenz kann man angesichts dieser Zahlen nicht sprechen.

Tabelle 2: Ausgewanderte Ärzte zwischen 2001 und 2007

\begin{tabular}{lccc}
\hline Jahr & $\begin{array}{c}\text { berufstätige } \\
\text { registrierte Ärzte }\end{array}$ & $\begin{array}{c}\text { ausgewanderte } \\
\text { Ärzte absolut }\end{array}$ & $\begin{array}{c}\text { ausgewanderte } \\
\text { Ärzte }- \text { Anteil }\end{array}$ \\
\hline 2001 & 297.893 & 1.437 & $0,48 \%$ \\
2002 & 301.060 & 1.691 & $0,56 \%$ \\
2003 & 304.117 & 1.992 & $0,66 \%$ \\
2004 & 306.435 & 2.731 & $0,89 \%$ \\
2005 & 307.577 & 2.249 & $0,73 \%$ \\
2006 & 311.230 & 2.575 & $0,82 \%$ \\
2007 & 314.912 & 2.439 & $0,77 \%$ \\
\hline
\end{tabular}

Quellen: BÄK, Ärztestatistik 2007, 2008, Kopetsch 2007, eigene Berechnung

Für deutsche Nachwuchswissenschaftler aller Disziplinen sind die USA das bei weitem wichtigste Zielland. Nach der Promotion forschen, nach Ergebnissen der TALENT-Studie (vgl. Buechtemann 2001), 12 bis 14\% für im Durchschnitt 3 bis 4 Jahre als ,Postdocs ${ }^{6}$ im Ausland. 37\% davon entfallen auf das Fach Medizin (ebd.: 4). Neben dem Erwerb von Wissen und Methodenkompetenz führt der Auslandsaufenthalt immerhin für etwa ein Viertel zur Habilitation. Da verlässliche Daten zum Rückkehrverhalten nicht vorliegen, gibt es lediglich Expertenschätzungen zu den tatsächlichen Abwanderungsquoten oder des „brain drain“. Angenommen wird, dass zirka ein Viertel bis ein Drittel der deutschen Postdocs längerfristig (aber nicht endgültig) in den USA bleiben. „Über dem Durchschnitt liegt die Verbleibquote in den Natur- und speziell den Biowissenschaften sowie der Physik, deutlich niedriger indes in der Medizin" (ebd.: 22). Dies entspricht, bezogen auf die Grundgesamtheit aller ca. 36.000 promovierten Nachwuchswissenschaftler in Deutschland und die etwa 5.000 bis 6.000 ,German Scholars' (im Jahr 2001, vgl. ebd.: 22) einem Ausmaß von maximal ca. 540 Personen. Buechtemann zufolge spricht dies dafür, dass die quantitative Relevanz von Abwanderungen eher überschätzt würde (ebd.: 22).

\subsection{Der medizinische „brain gain“ in Deutschland}

Aktuell stehen den etwa 19.000 deutschen Ärzten im Ausland über 20.000 ausländische Ärzte, von denen über 16.000 berufstätig sind, in Deutschland gegenüber. Betrachtet 
man also umgekehrt den „brain gain“ in Deutschland, so zeigen die verfügbaren Daten, dass die Einwanderung ausländischer Ärzte seit einem Höchststand im Jahr 2003 mit 1.971 Zugewanderten kontinuierlich abgenommen hat und dann im Jahr 2006 nur noch 1.404 betrug (Kopetsch 2007). Sie stieg allerdings im darauf folgenden Jahr 2007 wieder um fast 25\% auf 1.735 an (vgl. Kopetsch 2008). Sollte sich dieser längerfristige Trend bestätigen, dann wäre Deutschland in den letzen vier Jahren tatsächlich zu einem Ärzteexporteur geworden, wenn auch in einem sehr geringen Umfang.

Die Ärztestatistik der Bundesärztekammer (BÄK) geht für das Jahr 2007 insgesamt von 413.696 registrierten Ärztinnen und Ärzten in Deutschland aus. Darunter finden sich 20.434 ausländische Ärztinnen und Ärzte, davon sind 16.818 berufstätig (siehe Tabelle 3). Der überwiegende Anteil stammt aus Europa, wobei Österreich, Griechenland, Polen und Russland die größten „Exporteure“ sind. Die bemerkenswert hohe Anzahl von Ärzten iranischer Herkunft (1.106) stellt mit fast 28\% den Hauptanteil der Ausländer aus Asien dar. Es folgen Ärzte aus Syrien, Jordanien und Afghanistan.

Tabelle 3: Ausländische Ärztinnen und Ärzte in Deutschland 2007, gegliedert nach Tätigkeitsbereichen (Anzahl)

\begin{tabular}{lcccc}
\hline Herkunft & $\begin{array}{c}\text { ausländische } \\
\text { Ärzte gesamt }\end{array}$ & berufstätig & im Krankenhaus & niedergelassen \\
\hline EU & 10.069 & 8.516 & 6.047 & 1.828 \\
Übriges Europa & 4.557 & 3.732 & 2.806 & 567 \\
Asien & 3.994 & 3.094 & 2.112 & 228 \\
Afrika & 850 & 719 & 543 & 134 \\
Amerika & 710 & 562 & 374 & 116 \\
Sonstige & 254 & 195 & 120 & 55 \\
\hline
\end{tabular}

Quelle: BÄK, Ärztestatistik 2007

Bleibt der Blick auf die Anteile der ausländischen Ärzte an den Positionseliten in der Medizin: den Klinik- und abteilungsleitenden Chefärzten und Ärztlichen Direktoren in Deutschland. Die Betrachtung der Besetzungen von Spitzenpositionen in Krankenhäusern bietet hierfür einen guten Indikator. Nach Angaben der Bundesärztekammer liegen zwar Statistiken über die Anzahl und die Herkunft von ausländischen Krankenhausärzten vor, es gibt zentral jedoch keine Angaben zu den Positionen, die sie im Gefüge der organisierten Medizin einnehmen. Nach den Informationen der angefragten Landesärztekammern betragen im Krankenhaussektor die Anteile leitendender Ärzte und Chefärzte mit ausländischer Herkunft derzeit maximal 4,2\% (vgl. Tabelle 4). Da nur wenige Daten verfügbar sind und die Statistiken der Landesärztekammern uneinheitliche Differenzierungen vornehmen, kann zur Frage der medizinischen Positionselite nur einschränkend gesagt werden, dass im Durchschnitt leitende Positionen (Chefärzte, leitende Ärzte und ärztliche Direktoren) in deutschen Krankenhäusern lediglich zu 2,1\% nicht durch deutsche Mediziner besetzt werden. 
Tabelle 4: Anteil Ärzte in Spitzenpositionen im Krankenhaus mit ausländischer Herkunft, nach Angaben der Landesärztekammern (LÄK)

\begin{tabular}{lc}
\hline LÄK & Leitende Ärzte mit ausländischer Herkunft \\
\hline Bayern & $2,85 \%$ \\
Sachsen & $0,72 \%$ \\
Brandenburg & $2,71 \%$ \\
Nordrhein & $4,19 \%$ \\
Schleswig-Holstein & $0,00 \%$ \\
Nordbaden & $2,39 \%$ \\
\hline
\end{tabular}

Zur ,medizinischen Elite“ im engeren Sinne sind allerdings lediglich die deutschlandweit etwa 1.200 Chefärzte und Ärztlichen Direktoren der Universitätsklinken zu zählen. ${ }^{19}$ Über diese durch ihre herausgehobenen Positionen definierte Gruppe von Medizinern liegen ebenfalls nur spärlich Daten vor. ${ }^{20}$ Aus diesem Grund geben unsere Fallanalysen von drei Universitätsklinken ${ }^{21}$ (vgl. Tabelle 5) einen ersten Einblick in diese Frage.

Tabelle 5: Leitende Ärzte mit ausländischer Herkunft in drei deutschen Universitätsklinken

\begin{tabular}{lccc}
\hline & $\begin{array}{c}\text { Ärztliche Direktoren } \\
\text { und Abteilungsleiter }\end{array}$ & \multicolumn{2}{c}{ davon mit ausländischer Herkunft } \\
& 26 & 4 & Anteil (\%) \\
\hline Uni-Klinikum A & 20 & 0 & 15,4 \\
Uni-Klinikum B & 32 & 5 & 0,0 \\
Uni-Klinikum C & & & 15,6 \\
\hline
\end{tabular}

Betrachtet man, gemessen an diesen Fallanalysen, den „brain gain“, so liegt der Anteil der ausländischen Chefärzte und Ärztlichen Direktoren bei insgesamt 11,6\% - und damit deutlich höher als die auf Bundeslandebene über alle Krankenhäuser errechneten etwa 2 Prozent. Allerdings kommen von diesen neun Personen sechs aus dem überwiegend deutschsprachigen Kulturraum der Schweiz bzw. Österreichs.

\subsection{Nationale Karrieremuster und „Internationalisierung light“ in der Medizin}

Auch wenn wir in der Frage der Internationalität der Top-Mediziner in Deutschland nur erste Anhaltspunkte geben konnten, lassen die hier vorgestellten Ergebnisse insgesamt erkennen: Die gezeichneten Krisenszenarien einer bedrohten Funktionsfähigkeit des Gesundheitssystems lassen sich empirisch nicht rechtfertigen. Von einer „internationalen“ Arbeitsmigration von Medizinern in einem nennenswerten Umfang kann keine Rede sein. Entgegen den Annahmen der Globalisierungstheorie zeigt sich, dass der nationale Arbeitsmarkt für Ärzte eine steigende Beschäftigung, aber eine geringe Berufsmobilität über die Grenzen hinweg aufweist. Eine Vielzahl der vermeintlich ausgewanderten deutschen Ärzte nutzt die Auslandstätigkeit eher als Karrierekatalysator. Ärzte zählen - neben 
Ingenieuren und Kaufleuten (vgl. dazu auch Migrationsbericht 2005: 114) - eher zu den „temporären“ Abwanderern und nicht zu den ,klassischen“ Auswanderern. Wir können hier von einer „Internationalisierung light“ sprechen, weil ihre Karrierepfade nationalen Karrieremustern folgen und das Ausmaß von Karrieremustern durchbrechenden ,transnationalen Karrieren" sich in diesem Feld in engen Grenzen hält.

Die geringe Internationalisierung in Spitzenpositionen im Krankenhaus lässt sich am besten über die Karrieremechanismen und -logiken in der Medizin erklären. Zu unterscheiden sind drei typische Karrierepfade: erstens die Niederlassung nach einer Assistenztätigkeit im Krankenhaus, in der Regel verbunden mit einer Facharztausbildung, zweitens die Krankenhauskarriere, die über Assistenz-, Facharzt- und Oberarzttätigkeit zu einer Chefarztposition führt, und drittens die wissenschaftliche Karriere, die in Universitätsklinken im günstigsten Fall in eine leitende Position mündet. Für den ersten Fall der selbständigen Arztpraxis ist internationale Erfahrung nicht erforderlich, gefragt ist hier die Passförmigkeit der fachlichen Ausbildung zur angestrebten Niederlassungsregion. Auslandserfahrung schadet zwar nicht, verzögert aber unter Umständen den Eintritt in ein ohnehin durch Versorgungsquoten stark reguliertes Feld. In den Fällen einer Krankenhauskarriere spielt das jeweilige organisationale und institutionelle Umfeld die entscheidende Rolle. Für Chefarztpositionen in kleineren Krankenhäusern oder klinischen Abteilungen (in städtischen Klinken, Kreiskrankenhäusern, Reha-Kliniken usw.) steht als Anforderung die klinische Expertise ganz oben. Auslandserfahrung ist auch hier unschädlich, aber ebenso nicht erforderlich. Es zählt das Können und die Erfahrung im fachlichen Bereich der zu leitenden Klinik. Für leitende und höchste Positionen in Universitätsklinika ist internationale Erfahrung hingegen ein unbedingtes Erfordernis. In diesem Kontext spielen Auslandsaufenthalte zur wissenschaftlichen oder auch fachlichen Weiterbildung eine große Rolle. Die Karrieren werden - neben den wissenschaftlichen Leistungen - häufig durch Lehrer-Schüler-Verhältnisse geprägt, die auch für die Besetzung von Chefarztpositionen über Berufungen relevant sind. Da aber auch der „Inflow" ausländischer Mediziner oft zeitlich befristet ist und daher häufig nur zu einer begrenzten Relevanz von Lehrer-Schüler-Verhältnissen führt, sorgen diese Karrieremechanismen dafür, dass die internationale Erfahrung der Chefärzte zwar hoch ist, aber der Internationalisierungsgrad medizinischer Spitzenpositionen eher gering bleibt.

\section{Die Migration von Hochqualifizierten und Führungskräften in der Ökonomie}

Noch stärker als in der Medizin scheint die Globalisierung in der Privatwirtschaft fortgeschritten zu sein. Zumindest hat die Bedeutung von trans- oder multinationalen Unternehmen in den letzten Jahrzehnten sehr stark zugenommen. Dass diese von einem transnationalen Management geführt werden, erscheint vielen Autoren als ebenso selbstverständlich wie, dass dieses „transnationale Management“ auf internationalen Arbeitsmärkten heiß umworben ist. „Brain drain“ und „brain gain“ sind daher auch die gängigen Stichworte dieser Diskussion in den Medien und der Politik. Um auch in diesem Feld die Tragfähigkeit und Stichhaltigkeit dieser Beschreibungen zu prüfen, verbinden wir, wie auch zuvor, Sekundäranalysen von vorhandenen Statistiken mit eigenen Forschungsbefunden zum Top-Management in Deutschland. 
4.1 Wie international ist Deutschlands Management?

Einen ersten Zugang zur Frage des „,brain gain“ in Deutschland eröffnet die Beschäftigtenstatistik der Bundesagentur für Arbeit. Nach dieser waren 2005 mehr als 100.000 hochqualifizierte Ausländer mit einem Hochschulabschluss in Deutschland beschäftigt. Sie arbeiteten vor allem im Dienstleistungssektor - insbesondere in unternehmensorientierten Dienstleistungsunternehmen (22\% aller ausländischen Beschäftigten mit Hochschulabschluss), im Bildungsbereich (rund 16\%) sowie im Gesundheits-, Veterinär- und Sozialwesen (11\%). Im Verarbeitenden Gewerbe ließ sich ein Schwerpunkt bei der Herstellung von Büromaschinen und Datenverarbeitungsgeräten, Elektrotechnik, Feinmechanik und Optik (9\%) erkennen (vgl. IAB Kurzbericht 12/2007: 2 ff.). Genauere Ergebnisse zu den uns interessierenden Positionen liefert eine von uns durchgeführte Sonderauswertung des Mikrozensus zu den Positionsgruppen der ausländischen Geschäftsführer und Bereichsleiter, die in Deutschland tätig sind. Mit 6\% im Jahre 2004 und 7,3\% im Jahre 2006 ist der Anteil der ausländischen Unternehmer, Geschäftsführer und Bereichsleiter in Deutschland zwar gestiegen, aber bewegt sich - gemessen an den im Globalisierungsdiskurs formulierten Erwartungen - auf einem vergleichsweise geringen Niveau. Denn diese Statistik bezieht sowohl eine Vielzahl von Kleinunternehmern z. B. aus der Türkei oder Polen mit ein, als auch Führungskräfte, die zeitweise in ausländischen Klein- und Mittelunternehmen arbeiten oder deren Vertrieb übernommen haben. Eine weitere Unterscheidung nach Unternehmensgrößen oder Funktion ist im Mikrozensus nicht möglich.

Tabelle 6: Ausländische Unternehmer, Geschäftsführer und Bereichsleiter in Deutschland

\begin{tabular}{|c|c|c|c|c|}
\hline 2004 & $\mathrm{BRD}$ & davon Ausland & Europa & übrige Welt \\
\hline Unternehmer/Geschäftsführer & 643.000 & $6,5 \%(43.000)$ & $6,0 \%$ & $0,5 \%$ \\
\hline Bereichsleiter & 357.000 & $5 \% \quad(18.000)$ & $3,0 \%$ & $* \%$ \\
\hline Gesamt & $1,000.000$ & $6 \% \quad(60.000)$ & $5,1 \%$ & $0,9 \%$ \\
\hline \multicolumn{5}{|l|}{2006} \\
\hline Unternehmer/Geschäftsführer & 601.000 & $8,1 \%(49.000)$ & $6,6 \%$ & $1,5 \%$ \\
\hline Bereichsleiter & 369.000 & $5,9 \%(22.000)$ & $4,5 \%$ & $1,4 \%$ \\
\hline Gesamt & 970.000 & $7,3 \%(71.000)$ & $6,9 \%$ & $1,4 \%$ \\
\hline
\end{tabular}

* Aufgrund zu geringer Zahlenwerte ist keine Angabe möglich.

Quelle: Mikrozensus 2004; 2006 eigene Auswertungen

Auch in den mittleren und obersten Führungsetagen der Top-100-Unternehmen in Deutschland ändert sich dieses Bild nicht. Betrachtet man die Anteile von aus dem Ausland zugezogenen oder dort aufgewachsenen Führungskräften an allen Führungskräften an den deutschen Standorten der Konzerne, so liegt diese im Durchschnitt von 12 Unternehmensfallstudien, die wir durchgeführt haben, bei 5,5\% (vgl. Pohlmann 2009). Dies lässt sich durch die Ergebnisse anderer Studien bestätigen. So kommt der IZA-Report zur Nachfrage nach ausländischen Hochqualifizierten in deutschen Unternehmen zum 
Ergebnis, dass diese in Unternehmen, die überhaupt ausländische Hochqualifizierte beschäftigen (39\%), nur rund 9\% aller hochqualifizierten Beschäftigten der Unternehmen ausmachen (vgl. Winkelmann et al. 2001: 33). Der durchschnittliche Anteil unter allen befragten Unternehmen beträgt 3,5\%. Davon sind die weitaus meisten ausländischen Hochqualifizierten als Professionals bzw. Spezialisten in Forschung und Entwicklung, dem IT-Bereich sowie in Marketing und Vertrieb beschäftigt. Führungskräftepositionen nehmen nur wenige ein (vgl. ebd.: 67 ff.). Für das Top-Management in Deutschland weisen die Daten von Hartmann für 2005 mit neun im Ausland aufgewachsenen Top-Managern einen nur leicht höheren Prozentsatz auf (vgl. Hartmann 2007: 59). Zwar ist die Zahl der Ausländer unter den Vorstandsvorsitzenden der Top-100-Unternehmen von 1995 bis 2005 leicht gestiegen, aber auch hier kommen nicht wenige aus der benachbarten Schweiz oder aus Österreich. Jene Briten oder US-Amerikaner, so Hartmann, deren Einkommen in der Diskussion über Managergehälter stets als Vergleichsmaßstab herangezogen werden, finden sich an der Spitze der deutschen Top-Unternehmen nicht (Hartmann 2007: 59; vgl. dazu auch Pohlmann 2009).

\subsection{Die Abwanderung der ,,besten Köpfe“?}

Der Zugang zu Daten in Bezug auf den „,brain drain“ ist außerordentlich schwierig und erst recht, wenn es sich um Führungskräfte oder Top-Manager der Privatwirtschaft handelt. ${ }^{22}$ Zunächst einmal kann man mit der Cheers-Studie Ende der 90er Jahre darauf hinweisen, dass die Auslandstätigkeit deutscher Hochschulabsolventen vier Jahre nach ihrem Studienabschluß sich dieser Studie zufolge mit 2,3\% unterhalb des europäischen Durchschnittes von 2,7\% bewegte (Jahr et al. 2002: 28 f.). Die Mobilen waren deutlich häufiger in der Privatwirtschaft beschäftigt (59\% zu 42\%) und seltener im öffentlichen Dienst (27\% zu 42\%). Entsprechend dominierten bei den Mobilen Wirtschaftssektoren, die typischerweise privatwirtschaftlich organisiert sind, wie das produzierende Gewerbe (39\% zu 24\%), während die Bereiche öffentliche Verwaltung und Gesundheitswesen unterdurchschnittlich vertreten waren (Jahr et al. 2002: 49). Enders/Bornmann (2002a) haben darüber hinaus die internationale Mobilität von bundesdeutschen promovierten Akademiker/innen dreier Abschlussjahrgänge in sechs Fächergruppen untersucht. Sie kommen zu dem Ergebnis, dass mit fachlichen Unterschieden, jeder Vierte bis Sechste nach der Promotion ins Ausland geht, dieser Aufenthalt aber zumeist intermediär bleibt (ebd.: 358). Etwa ein Viertel der Biologen, Mathematiker und auch der Wirtschaftswissenschaftler sind demnach nach der Promotion im Verlauf ihrer Karriere im Ausland tätig gewesen (Enders/Bornmann 2002b: 62). Enders/Mugabushaka (2004) hatten Stipendiaten der DFG im Blick. Hier zeigt sich, dass die Quote der nach Ablauf des Stipendiums im Ausland Verbleibenden mit disziplinären Schwankungen zwischen 0 und 19\% beträgt. ${ }^{23}$ Im Umkehrschluss heißt das, dass über $80 \%$ wieder nach Deutschland zurückkehren. Diehl/Dixon (2005) untersuchten die Auswanderung Hochqualifizierter in die USA und kommen zu dem Ergebnis, dass sich, absolut betrachtet, nur wenige Deutsche dauerhaft zu Erwerbszwecken in den USA niederlassen und zeitlich befristete US-Aufenthalte überwiegen (Diehl/Dixon 2005: 714 ff.). Schupp et al. (2005) haben auf der Basis eine Längsschnittauswertung des SOEP mittels Weiterverfolgungskonzept soziode- 
mographische Angaben zu 280 Auswanderern untersucht. Sie stellen fest, dass Hochqualifizierte häufiger vertreten sind, sie aber absolut betrachtet nicht die Mehrzahl darstellen. Die Auswanderer stammen zu zwei Dritteln aus der Gruppe der jüngeren Personen unter 35 Jahren und Frauen sind überrepräsentiert (ebd.: 289).

Bezogen auf das Top-Management in verschiedenen OECD-Ländern zeigen unsere Daten zu den CEO der Top-100 (Industrie-)Unternehmen in Japan, Südkorea, China und Indien zwischen 2005 und 2008, dass unter diesen keine deutschen Manager vertreten sind (vgl. Pohlmann 2009). Dasselbe Bild zeigt sich auch in Großbritannien, Frankreich und den USA. Nach den Daten von Hartmann hält sich auch hier die „brain-drain“-Dynamik auf den Spitzenposition der Wirtschaft in engen Grenzen (vgl. Hartmann 2007). Die Rede von einem transnationalen Management oder einer globalen Elite erscheint vor diesem Hintergrund jedenfalls kaum gerechtfertigt.

\subsection{Karrieremuster in der Industrie: Insider und Outsider}

Dabei fällt zunächst auf, dass die Unternehmen zwar international erfahrenes Personal bevorzugen, aber dies umso mehr, wenn die Erfahrungen im Kontext der jeweiligen Unternehmensgruppe gesammelt wurden. Entsprechend zeigen unsere Ergebnisse zu den Unternehmens- und Branchenwechsel der Top-Manager, dass sich diese in engen Grenzen halten und nach wie vor Insider bevorzugt rekrutiert werden (vgl. dazu ausführlich Pohlmann 2009). Dass dies weltweit die Tendenz ist, zeigen nicht nur unsere TopManager-Studien in Ostasien, den USA und Indien, sondern auch die CEO-Nachfolgestudie von Booth, Allen und Hamilton bei 2.500 börsennotierten Unternehmen. Dieser zufolge stieg der Outsider-Anteil unter den $\mathrm{CEO}^{24}$ im weltweiten Durchschnitt von 14\% im Jahr 1995 zwar auf 30\% im Jahr 2003, aber sank 2006 wieder auf 18\% (Lucier et al. 2007: 6; vgl. dazu auch ausführlich Pohlmann 2009). Solange also „Insiderkarrieren“ in den Großunternehmen der führenden Industrienationen eher die Regel als die Ausnahme darstellen, beschränkt dies auch die globale Karrieremobilität des Personals. Denn deren Handlungsorientierungen werden zu einem erheblichen Maße durch die organisationalen Karrieresysteme geprägt, aber umgekehrt natürlich auch durch die Orientierungen des Personals.

Wir können anhand der von uns durchgeführten 89 Top-Manager-Interviews zeigen, warum die Karrieren in der Regel nicht im Ausland fortgesetzt werden und welche Faktoren dafür verantwortlich zeichnen.

So zeigt sich, dass für die Manager zwar Auslandsaufenthalte wichtig sind, aber als Teil einer in Deutschland absolvierten Karriere. Für viele ist es eine Karrierestation mit spezifischen Herausforderungen und der Erweiterung ihres Arbeitsgebietes, aber kein Wechsel des unternehmensbezogenen oder gar nationalen Karrierepfades. Exemplarisch dafür kann folgendes Interviewzitat eines Top-Managers (TM 28) stehen, der darlegt, dass sein Ausgangspunkt und ,sicherer Hafen“ immer in Deutschland geblieben ist.

„.. . und ich war ja, lieber Gott, ich war dauernd im Ausland. ' 85 war ich, weil ich technischer Chef der [Firmenname] war in New York, aber auch technischer Chef von [Firmenname] Europe - also ich habe den Job nicht abgegeben, hab mir selber berichtet - und Vorstandsmitglied, 
Technischer Direktor hier - einfach mit der Maßgabe: es kann nie schaden, einen deutschen Vorstandsvertrag zu haben, das ist immer was wert - aber ich musste eben alle drei Jobs ausfüllen, war ich in einem Jahr 53-mal in New York." (TM 28)

Diese Form einer Risikobegrenzung durch Beibehaltung der Vorstandsposition in Deutschland ist typisch für die Top-Manager-Karrieren. Diese werden auch von den deutschen Managern in der Regel unternehmensbezogen gedacht und angelegt. Der Rest wird einer oft lebensweltlich begrenzten Entsendedynamik überlassen, die eine Fortsetzung der Karriere im Ausland von vorneherein restringiert. Für die Beurteilung der eigenen Karriereentwicklung stehen Stationen im Ausland daher auch eher für die Ausdehnung der Zuständigkeit - im geographischen Sinne wie auf die Menge des nachgeordneten Personals bezogen - und werden in der Darstellung der Karriere neben der Geschwindigkeit des Aufstiegs zur Relevanzproduktion genutzt.

Sowohl für die jüngeren als auch für die älteren Top-Manager in unserer Untersuchungen sind längere Auslandsaufenthalte daher eine Form der Bewährung für die Inlands-, zumeist auch die Inhouse-Karriere. So berichten zwei der Top-Manager:

„Da war ich dann stellvertretender Leiter dieses Bereichs und hatte dann eben direkten Kontakt
mit der Geschäftsführung und dann haben sie mir die USA angeboten. Dann war ich für 4 Jahre
in den USA und habe in einer stürmischen Wachstumsphase den Umsatz wieder von 250 Mil-
lionen auf eine Milliarde innerhalb von 4 Jahren gesteigert. Ich war für 2 Werke zuständig.
(...) Als ich dann zurückkam, wurde ich Nachfolger des Bereichsleiters, der mich eingestellt
hat, der ging dann in Ruhestand, damit hat der Kreis sich geschlossen. Ich wurde dann wenige
Jahre später Vorsitzender der Geschäftsführung.“ (TM 47)

„Und als ich dann bei dem Unternehmen war, kam dann nach zwei Jahren die Frage an mich, ob ich bereit sei, in die USA zu gehen. Und den Ball habe ich sofort aufgefangen, weil ich mir gesagt habe, Amerika sowieso, damals gab's noch keine antiamerikanischen Tendenzen. Das war also sozusagen das Land der großen Verheißungen. Und dann war es natürlich für eine Karriere in einem multinationalen Unternehmen sicherlich ein Schritt, der nicht der letzte sein würde. Man war ja schon mal ausgesucht, wenn man so ne Führungsverantwortung übernehmen sollte.“(TM 58)

Hinzu kommt, dass auch die Karrieresysteme international ausgerichteter Unternehmen sich in der Nachwuchsrekrutierung an der Herkunft und die Karrieremobilität an der Bewährung und der Sozialisation im Unternehmen ausrichten und insofern faktisch „Grenzen“ für die internationale Mobilität des Personals schaffen und reproduzieren. Aufgrund der zeitlichen Befristung der Auslandsengagements eröffnen sich unseren Fallstudien zufolge auch für Impatriates aus dem Ausland nicht dieselben Karriereoptionen wie für Inländer. Auch für diese ist deswegen das dominante Karrieremuster die zeitlich begrenzte Entsendung, eine Art „Internationalisierung light“, und nicht die durchgängige Internationalisierung der Karriereverläufe. Mit „Internationalisierung light" ist dabei auch hier gemeint, dass die internationalen Rekrutierungsformen und mechanismen die national und organisational geprägten Karrieresysteme nicht durchbrechen, sondern die Internationalisierung des hochqualifizierten Personals ganz im Gegenteil deren Regeln folgt. Deswegen werden die oft zitierten, möglicherweise höheren Verdienstchancen im Ausland für die Akteure durch das Risiko von Karrieren- 
achteilen überschattet (vgl. zu dieser Diskussion auch: Mense-Petermann/Wagner 2006; Wagner 2006; Klemm/Popp 2006). Dies schlägt sich unseren Interviews zufolge in deren Handlungsorientierungen klar nieder. Ein anderer Top-Manager (TM 36) verweist vor diesem Hintergrund auf die klaren Grenzen, welche auch Karrierestationen in den USA besitzen.

„.. . und ich war in Amerika und da habe ich eigentlich gesehen, zumindestens zum damaligen Zeitpunkt, ich war Senior Vice President, den Level erreicht hatte, den ein Deutscher da erreichen konnte. Und, und das war eigentlich, war ja, war irgendwie so das Ende einer Entwicklung und das hat mich bewogen zu sagen: ,Da bleibe ich nicht, ich bleibe nicht auf dem Level in Amerika hängen.' Und dann bin ich zurückgekommen nach, nach Deutschland, weil ich ein Angebot gekriegt habe als Vorstandsvorsitzender.“ (TM 36)

Deutlich wird hier, was sich auch in anderen Interviews zeigt: Die Karrieren für Manager mit Migrationshintergrund enden auch in den multinationalen Großunternehmen vor dem Erreichen der Top-Führungspositionen. Dafür zeichnen die ungeschriebenen Regeln der Karrieresysteme verantwortlich, die Herkunft, Bewährung und Sozialisation zu „Dunkelfaktoren“ einer Beförderung (Bosetzky) in die höchsten Etagen werden lassen, die für „Outsider“ kaum überwindbare Barrieren schaffen.

„Das charakterisiert [Firmenname] und das habe ich erlebt, und das war auch eine Sache, die mich am Schluss, je höher man kommt, zunehmend frustriert hat." (TM 36 über seine Zeit in den USA)

Unsere Top-Manager-Interviews zeigen daher deutlich, dass die bisweilen angeführten „Trabanten-Karrieren“, bei denen eine Rückkehr in das Heimatland also entweder nicht gesichert oder gar nicht gewünscht ist, im deutschen Top-Management selten sind. Auslandserfahrung wird zwar geschätzt, ja im mittleren Management erwartet, aber:

„Die Realität ist inzwischen so, dass man gar nicht mehr mit der Gewissheit zusagen kann, dass es nach der Auslandsstation hier in Deutschland wieder 'ne Position gibt.“ (TM 31)

So haben zwar die Auslandsaktivitäten bei der jüngeren Generation des Top-Managements stark zugenommen (siehe Pohlmann 2009), aber auch ihre Karrieren bleiben durchgängig im deutschen Mutterunternehmen verankert. Nicht die Fortsetzung der Karriere im Ausland, sondern eine temporäre Entsendung nach dem Prinzip einer „Internationalisierung light" ist aufgrund der Karrieresysteme das gängige Muster (vgl. für das mittlere Management auch Faust 2002; Kotthoff/Wagner 2008). Dafür hat die Globalisierung der Unternehmen gesorgt, ohne die zentralen Karrieremechanismen jedoch zu verändern. Denn die Karrieren von Top-Managern stellen sich als abhängig von den „Karrierepfaden“ und internen Karrierenetzwerken eines Unternehmens dar - gerade auch dann, wenn längere Phasen davon im Ausland verbracht wurden. Daran richten sich auch die Handlungsorientierungen der Akteure aus, denen sich in einem ebenfalls von Hauskarrieren dominierten Ausland keine weiterreichenden Karriereoptionen eröffnen. 


\section{Globalisierung als „Internationalisierung light" und die Persistenz organisationaler Karrieremechanismen - ein Fazit}

Im Zuge der Globalisierung erhöht sich die weltweite Mobilität und Verfügbarkeit des Personals. Dabei gilt: Je qualifizierter das Personal, desto mobiler ist es. Für Studierende, angehende Führungskräfte, hoch qualifizierte ,professionals“ und „high potentials“ gehören Auslandsaufenthalte zum, guten Ton“ in der Gestaltung und Darstellung des eigenen Karriereweges. Dennoch kontrastieren damit die Anteile derjenigen, die ihre Karriere im Ausland fortsetzen. Eine „starke“ Globalisierungsthese, die davon ausgeht, dass diese zu einer internationalen Verteilung des hochqualifizierten Personals und zu übernationalen Regulierungsstrukturen (im Sinne von Verteilungsmechanismen, wie z. B. internationale Arbeitsmärkte, Netzwerke oder globale Verteilungskoalitionen) führe, muss zumindest anhand unserer Daten zurückgewiesen werden. Die Internationalisierung findet viel eher in Form einer Entsendedynamik zwischen Organisationen und mittels temporärer Auslandsaufenthalte statt, aber nicht als eine weitergehende Form der Transnationalisierung von Karrieren. „Brain circulation“ und nicht „brain drain“ ist das dominante Muster (siehe dazu ausführlich für die ökonomischen Eliten in Ostasien, den USA und Deutschland Pohlmann 2009). Natürlich ergeben sich daraus Verflechtungszusammenhänge, die man unter dem Stichwort der „Transnationalisierung“ der sozialen Welt untersuchen kann (vgl. dazu Pries 2008: 48 ff.). Aber eine - als Fortsetzung der Karrieren im Ausland verstandene - „Transnationalisierung“ der Karrierewege findet sowohl in der Medizin als auch in der Ökonomie nur in geringem Maße statt. Wenn wir dies als „Internationalisierung light“ bezeichnet haben, so ist damit gemeint, dass die nationalen und organisationalen Karrieresysteme die internationale Mobilität des hochqualifizierten Personals nach wie vor ganz maßgeblich prägen und den internationalen Markt- und Karrieremechanismen ihre Struktur geben - und nicht umgekehrt. Die Gründe dafür sind nach unserer Annahme weder allein in den Handlungsrationalitäten der Akteure noch den institutionellen Settings zu suchen, sondern in ihrem Zusammenwirken auf der Ebene der Organisation.

So bevorzugen Organisationen auf höheren Positionen bewährtes Personal - und zeigen damit eine - nach Luhmann typische - Präferenz für selbstgemachte Risiken (Luhmann 2000). Diese Präferenz für Insider wird gerne rein ökonomisch, also z. B. mit einer wechselseitigen Bindung durch Investitionen in das Humankapital, begründet (vgl. dazu Williamson 1975: 77; Köhler et al. 2007: 6 ff.). Aus organisationssoziologischer Sicht lässt sich jedoch darüber hinaus zum einen erkennen, dass sich mit ihr zugleich organisationale und soziale Rationalitäten gegenüber rein ökonomischen einfacher durchsetzen können. So zeigen empirische Befunde, dass Unternehmen mit intern rekrutierten Top-Managern („Insider“) auch dann stärker an Bestand und Wachstum orientiert sind, wenn betriebswirtschaftliche Kalküle eher für einen Verkauf von zugehörigen Betrieben oder Betriebsteilen sprechen würden. Mit Insidern erweist sich die Organisation in der Regel besser vor den Verkaufsabsichten von Aktionären und Investoren geschützt als mit extern rekrutierten Führungskräften (Lucier et al. 2007). Zum anderen kommen in den Karrieresystemen der Organisationen nicht proklamierte oder niedergelegte Dunkelfaktoren der Beförderung zur Geltung, welche Herkunft, Bewährung und Sozialisation zu wichtigen Kriterien der Rekrutierung für höhere und höchste Positio- 
nen werden lassen. Dies findet u. E. seinen Grund darin, dass Organisationen ihre Formalstrukturen nicht so ausbilden und ihre Entscheidungsprämissen nicht so setzen können, dass es Regeln und Vorschriften für alle Fälle gibt oder dass deren Befolgung in allen Fällen zweckdienlich ist. Da die Organisation nicht alles formal regeln kann, was in Organisationen legitimerweise stattfinden kann und soll, ist sie auf die Reproduktion von Wertorientierungen verwiesen, die sich durch interne Karrieren - also mittels organisationaler Bewährung und Sozialisation - am besten sicherstellen lässt. $\mathrm{Zu}$ diesen Wertorientierungen gehören unausgesprochene Regeln, welche Abweichungen von den formalen Regeln in einer Organisation legitim sind, welche Geltungskraft hintergründigen Orientierungen an sozialem Status, Alter oder Geschlecht in der Organisation eingeräumt wird, welche Art von Machtausübung oder Einflussnahme abseits der formalen Hierarchie akzeptiert wird etc. Es dreht sich um die Wertung und Normierung dessen, was hinter der Fassade formaler Rationalität, die sich die Organisation aus gesellschaftlichen Legitimitätserwartungen heraus gibt, zur Geltung kommt und neben Kalkülen die Handlungsorientierungen der Akteure bestimmt - seien sie im Weber'schen Sinne wertrational, affektual oder im Bourdieu'schen Sinne habitual orientiert. Jede Organisation ist auf die Bewährung und Sozialisation der Akteure in ihrer „Kultur“ verwiesen, da diese maßgeblich den Umgang mit Formalität und mit den nicht formellen Anforderungen aus den Umwelten der Organisation prägt. Denn nur die Kultur einer Organisation generiert und reproduziert jene hintergründigen Wertorientierungen und Handlungsschemata, welche auch der Handhabung ihrer ungeschriebenen Regeln als bewährte Praxis kollektive Geltung verschafft.

Für die Akteure selbst erhöhen sich damit - entgegen den Annahmen der Globalisierungstheorie und der Politik bezüglich der Entstehung eines verschärften internationalen Wettbewerbs „um die besten Köpfe“ - die Risiken von zu langen Auslandsaufenthalten oder Auslandskarrieren. Aufgrund der organisationalen Karrieresysteme fällt es ihnen schwer, ihre Karriere im Ausland fortzusetzen. Zugleich riskieren sie, im Inland von den Karrierenetzwerken in ihrer Organisation abgekoppelt zu werden. Und je länger der Auslandsaufenthalt dauert, desto größer erscheint dieses Risiko. Erst in der Analyse des Zusammenspiels dieser Handlungsorientierungen mit den organisationalen Karrieremechanismen lassen sich die Hintergründe für die Grenzen der internationalen Mobilität des hochqualifizierten Personals klarer erkennen.

\section{Anmerkungen}

1 Unser Verständnis von Hochqualifizierten ist ein formales: Wir bezeichnen damit Personen, die einen Hochschulabschluss bzw. einen ,univesity degree“ im Rahmen der „tertiary education“ (in Deutschland Fachhochschulen, Hochschulen und Universitäten) vorweisen können (vgl. zu einer etwas weiteren Definition auch Campbell/Rozsnyai, 2002: 133). Hochqualifiziert in unserem Zusammenhang bedeutet also: Erster oder zweiter Hochschulabschluss oder vergleichbare Ausbildung (entspricht den Gruppen 5 und 6 der ISCED, International Standard Classification of Education; vgl. dazu auch Jahr et al. 2002: 13).

2 So schreibt Chalamwong: „In recent years many OECD countries have increasingly adopted policies that promote the entry of skilled and highly skilled workers, especially in information and communication technology (Chalamwong 2005: 488). 
3 Ein einfacher Zugang zur Frage der internationalen Mobilität von Hochqualifizierten und Eliten besteht nach wie vor nicht, da insbesondere auch die Entsendedynamik zwischen den Unternehmen nicht hinreichend erfasst wird (vgl. für eine zusammenfassende Darstellung dieser Problematik auch Jahr et al. 2002: 8 f.).

4 Auch in Bezug auf die Definition von Eliten kommt hier der formale Positionsansatz zur Anwendung. Unter den ökonomischen Eliten verstehen wir die Vorstände und Vorstandsvorsitzenden der Top 100 bis Top 500 Großunternehmen in Deutschland, gelistet nach Umsatz und Beschäftigung (vgl. dazu auch Pohlmann 1997; 2002; 2009).

5 Anlässlich einer Anfrage im Bundestag zum Thema: Konsequenzen der Auswanderung Hochqualifizierter aus Deutschland wird Wolfgang Schäuble mit den Worten zitiert: „Im Übrigen stimmt die Bundesregierung mit den Fragestellern überein, dass es im Zuge der Globalisierung von Wirtschaft und Wissenschaft zunehmend zu einem Wettbewerb um die besten Köpfe kommt. Das ist ganz zwangsläufig so. Wir müssen aber darauf achten, in diesem Wettbewerb attraktiv zu bleiben." siehe http://www.bmi.bund.de/cln_012/nn_1136722/Internet/Content/ Nachrichten/Reden/2007/10/Rede_BM_Auswanderung_Hochqualifizierter.html, 13.05. 2008.

6 Bei der Vielfalt der Autoren und Schriften ist der Mainstream schwer zu erfassen und stellt hier eher eine Hilfskonstruktion dar, um die Annahmen der Globalisierungstheorien besser greifbar zu machen. Ich rechne zu diesem Mainstream der internationalen Globalisierungsdiskussion zumindest drei verschiedene Ansätze oder Perspektiven, die diese Diskussion in den letzten Jahren sehr stark geprägt haben. Erstens sind hier epochale Globalisierungstheorien zu nennen, wie sie von Albrow (1996; 1998), Beck (1997), Giddens (1990; 1994), Münch (2001), Featherstone (1990, 1995) u. a. entwickelt wurden, die davon ausgehen, dass mit der forcierten Globalisierung ein qualitativer Sprung in eine neue Epoche gemacht wurde, welche die (erste, nicht globale) Moderne ablöst. Globalität stellt sich dieser Literatur zufolge zwar immer wieder durch Globalisierung her, sie stellt aber keine Form der Vollrealisation einer Weltgesellschaft dar. Dasselbe gilt für die inkrementalen Globalisierungstheorien, wie sie von Barber (1995), Ohmae (1995), Robertson (1998) u. a. entwickelt wurden. Hier wird der Prozess im Wechselspiel von Globalisierung und Lokalisierung, von Homogenisierung und Heterogenisierung analysiert, quasi als endloser Prozess in einer sich gleichzeitig globalisierenden und fragmentierenden Welt. Neben diesen Ansätzen ist der Mainstream der Globalisierungsliteratur auch durch eher normative Bezugnahmen gekennzeichnet.

7 Der Präsident der Bundesärztekammer Hoppe geht in seinem Statement auf der Pressekonferenz am 9. Oktober 2007 bereits von einem solchen Mangel aus.

8 Appleyard (1995) spricht in diesem Zusammenhang von der Gruppe der „forgotten migrants“, und Hunger/Thränhard (2000) betonen, dass in der Migrationsforschung die Migranten meist als ausgebeutete, unterdrückte defizitäre Wesen vorkämen, die der Betreuung bedürften. Auch für Bommes (2006) werden viele Fragestellungen der Migrationssoziologie eher aus einem politischen, denn aus einem wissenschaftlichen Rahmen heraus formuliert (vgl. dazu auch Kolb 2006: 160).

9 Das Besondere an der Migrationsdynamik des 21. Jahrhunderts sei aber auch hier, Pries zufolge, ,dass aufgrund qualitativ verbesserter und breiten Bevölkerungsschichten zugänglicher Kommunikations- und Transportmöglichkeiten internationale Migrationsprozesse immer komplexer werden und immer weniger dem klassischen Bild der Ein-/Auswanderung oder der Rückkehrwanderung entsprechen" (Pries 2005a: 3).

10 Brücker geht davon aus (2007: 5, unter Hinweis auf: Brücker, H./J. von Weizsäcker [2007]: Migration Policy: At the Nexus of Internal and External Migration, in: Sapir, A. [Hg.]: Europe in the Global Economy, Brüssel: Bruegel, 226-265), dass die Wanderung von Hochqualifizierten in den OECD-Staaten beständig zunimmt. Davon betroffen seien die Abwanderung von Deutschen ins Ausland und die Zuwanderung aus der EU-15 und aus Nordamerika 
nach Deutschland gleichermaßen. Obwohl Deutschland damit einen Nettogewinn an qualifizierten Zuwanderern aus den entwickelten Mitgliedstaaten der OECD erzielen würde, seien die Gesamtzahlen so gering, dass sich dadurch kein nennenswerter Zuwachs an hochqualifizierten Fachkräften ergäbe. Anhand der Veränderungen der Auswanderungsrate deutscher Staatsangehöriger lässt sich erkennen, dass die Altersgruppen der 18- bis 25-Jährigen und die der 25- bis 40-Jährigen deutlich höhere Emigrationsraten als die Gesamtbevölkerung aufweisen. Diese betrug in den 1980er Jahren 1,5 bis 2 Personen pro 1.000 Einwohner gegenüber 2,91 der Altersgruppe der 18- bis 25-Jähringen sowie 3,83 pro Tausend der Altersgruppe der 25- bis 40-Jährigen im Jahre 2005 (Sauer, Ette 2007: 39).

11 Vgl.: http://www.auswaertiges-amt.de/diplo/de/Europa/DeutschlandInEuropa/BilateraleBeziehungen/Polen/RegGreZusammenarbeit/GrenzZusamm.html am 02.06.2008.

12 Gemäß der Daten des PSID. PSID ist die Abkürzung für Panel Study of Income Dynamics des Institute for Social Research der University of Michigan, Ann Arbor. Sie ist die Vorbildstudie für die Entwicklung des SOEP, weswegen ein hohes Maß an Vergleichbarkeit gegeben ist (Büchel et al. 2002: 211).

13 Das Sozioökonomische Panel (SOEP) ist eine repräsentative Wiederholungsbefragung, die auf der Haushaltsebene basiert und im jährlichen Rhythmus seit 1984 bei denselben Personen und Familien in der Bundesrepublik durchgeführt wird. In diesem Survey werden für die Forschung Mikrodaten bereitstellt.

14 Differenziert nach Abschlussarten sind es 2.290 an einer Universität (+253 an einer Hochschule in Ostdeutschland), 1.394 an einer FH (+315 an einer Fachschule/Ingenieurschule in Ostdeutschland) und 83 Abschlüsse an einer ausländischen Hochschule.

15 Aus der Differenz der Einzeldaten lassen sich Quoten von 15,1-29,1\% errechnen (vgl. Kopetsch 2008).

16 Die Statistiken (Kopetsch 2007, 2008) beruhen auf Angaben unterschiedlicher Jahre (2001-2007) und unterschiedlichen Quellen.

17 Es ist zu vermuten, dass die Schweiz als Zielland an Bedeutung gewinnen wird, da dort die institutionelle „Inländerbevorzugung“ mit einer schrittweisen Umsetzung des Personenfreizügigkeitsabkommen weggefallen ist (vgl. Schweizer Bundesamt für Migration (BFM), http:// www.bfm.admin.ch/bfm/de/home/themen/schweiz___eu.html, 13.01.2009).

18 Die Abwanderung nach Großbritannien nimmt deutlich ab, da die zentrale Vergabe der Weiterbildungsplätze nach Umstellung der Facharztausbildung (MMC: Modernizing Medical Careers) im Jahr 2007 ein Nadelöhr darstellt. „Hauptproblem ist, dass die ohnehin knappen Plätze bevorzugt an Briten vergeben werden. Erst dann kommen andere Europäer. Zudem strömen in Großbritannien immer mehr junge Ärzte auf den Arbeitsmarkt - unter anderem, weil neue medizinische Fakultäten gegründet wurden. Offensichtlich haben die Briten ihren Medizinermangel also erfolgreich bekämpft. Das ist erfreulich für das englische Gesundheitssystem - und schade für auswanderungswillige Mitteleuropäer.“ (Fritz 2007: http:// www.thieme.de/viamedici/laender/england/weiterbildung.html, am 02.02.2009).

19 Nach Schätzungen der Süddeutschen Zeitung, http://sueddeutsche.de/jobkarriere/38/ 339883/text, am 29.01.2009.

20 Wie viele Spitzenpositionen an den Universitätsklinken von Ärzten ausländischer Herkunft besetzt werden, lässt sich lediglich in Sachsen angeben, dort sind es von insgesamt 627 Positionen leitender Ärzte und Chefärzte an allen Klinikformen drei an Universitätsklinken.

21 Durchgeführt mittels Internetrecherchen.

22 Die Datenlage zur Migration von Fach- und Führungskräften in Deutschland ist unübersichtlich und schwer erschließbar. Insbesondere die Frage des Brain-Drain lässt sich nicht umfassend beantworten. Weder zur zeitweiligen noch zur dauerhaften Abwanderung deutscher Fach- und Führungskräfte liegen gesicherte Angaben vor, mit welchen der faktische Umfang beziffert werden könnte (Backhaus et. al. 2002: 1). 
$23,19 \%$ der Befragten aus den Naturwissenschaften leben heute im Ausland. Im Bereich Biologie/Medizin sind es 17\%, in den Geistes- und Sozialwissenschaften beträgt der Anteil 14\%. Aus den Ingenieurwissenschaften gab kein Befragter an, heute im Ausland tätig zu sein.“ (Enders/Mugabushaka 2004: 43).

24 Bemessen an den CEO, die ihren Abschied nahmen (Lucier et al. 2007: 6).

\section{Literatur}

Albrow, Martin. 1996. The global age, state and society beyond modernity. Cambridge: Polity Press.

Albrow, Martin. 1998. Abschied vom Nationalstaat, Staat und Gesellschaft im globalen Zeitalter. Frankfurt am Main: Suhrkamp.

Appadurai, Arjun. 1998. Globale ethnische Räume. Bemerkungen und Fragen zur Entwicklung einer transnationalen Anthropologie. In: Perspektiven der Weltgesellschaft, hrsg. Ulrich Beck, 11-39. Frankfurt/M.: Suhrkamp.

Appleyard, Reginald. 1995. New Trends in Migration. Numbers, Directions and Dynamics, Paper presented to the Conference „New Trends in Migration. Multiculturalism and beyond“. London.

Backhaus, Beate, Lars Ninke und Albert Over. 2002. Brain Drain - Brain Gain. Eine Untersuchung über internationale Berufskarrieren. Stifterverband für die deutsche Wissenschaft.

Barber, Benjamin R. 1995. Jihad vs. McWorld. New York: Times Books.

Bartlett, Christopher A., and Sumantra Ghoshal. 2001. Managing across borders, the transnational solution. 2. Aufl. London:Random House Business Books.

Beck, Ulrich. 1997. Was ist Globalisierung? Frankfurt/M.: Suhrkamp.

Bellemann, Lutz und Johannes Velling. 2002. Arbeitsmärkte für Hochqualifizierte. Beiträge zur Arbeitsmarkt und Berufsforschung, Beitrag 256. Nürnberg: IAB der Bundesanstalt für Arbeit.

Bommes, Michael. 2006. Migration and Migration Research in Germany. In: International Migration and the Social Sciences, hrsg. Ellie Vasta und Vasodeeven Vuddamalay, 143-221. Basingstoke: Palgrave Macmillan.

Brücker, Herbert. 2007. Migration als Therapie für Fachkräftemangel?. In: Fachkräftebedarf der Wirtschaft, Materialsammlung $C$, hrsg. Institut für Arbeitsmarkt- und Berufsforschung. Nürnberg: Ansatzpunkte für Therapien.

Buechtemann, Christoph F. 2001. Deutsche Nachwuchswissenschaftler in den USA. Perspektiven der Hochschul- und Wissenschaftspolitik. Projekt TALENT, hrsg. Bundesministerium für Bildung und Forschung. Bonn. pdf-File auf www.bmbf.de/pub am 26.06.2008.

Büchel, Felix, Joachim R. Frick und James C. Witte. 2002. Regionale und berufliche Mobilität von Hochqualifizierten. Ein Vergleich Deutschland USA. In: Arbeitsmärkte für Hochqualifizierte. Beiträge zur Arbeitsmarkt und Berufsforschung, hrsg. Johannes Vellingun und Lutz Bellemann, Beitrag 256. Nürnberg: IAB der Bundesanstalt für Arbeit.

Bundesärztekammer. 2007. Ärztestatistik der Bundesärztekammer 2007. http://www.baek.de am 29.12.2008.

Bundesministerium für Bildung und Forschung (BMBF). 2005. Internationalisierung des Studiums. Ausländische Studierende in Deutschland, hrsg. Deutsche Studierende im Ausland. Bonn, Berlin.

Campbell, C., and C. Rozsnyai. 2002. Quality Assurance and the Development of Course Programmes. Papers on Higher Education Regional University Network on Governance and Management of Higher Education in South East Europe Bucharest. UNESCO.

Castels, S.; M.J. Miller. 1993. The age of migration: international population movements in the modern world. London: Macmillan. 
Chalamwong, Yongyuth. 2005. The Migration of Highly Skilled Asian Workers to OECD Member Countries and its Effects on Economic Development in East Asia. In: Policy Coherence Towards East Asia, Development Challenges für OECD Countries. Development Center Studies. $O E C D$, hrsg. Alexandra Trzeciak-Duval, 487-526. Paris.

Diehl, Claudia und David Dixon. 2005. Zieht es die Besten fort? Ausmaß und Formen der Abwanderung deutscher Hochqualifizierter in die USA. Kölner Zeitschrift für Soziologie und Sozialpsychologie 57: 714-734.

Dreher, Sabine. 2003. Vom Wohlfahrtsstaat zum Wettbewerbsstaat? Die Bedeutung der Migration für die Globalisierungsdebatte. In: Migration im Wettbewerbsstaat, hrsg. Bernhard Santel und Uwe Hunger, 13-32. Opladen: Leske + Budrich.

Enders, Jürgen und Lutz Bornmann. 2002a. Internationale Mobilität von bundesdeutschen Promovierten. In: Arbeitsmärkte für Hochschulqualifizierte, hrsg. Johannes Velling und Lutz Bellmann, 357-374. Nürnberg: Institut für Arbeitsmarkt- und Berufsforschung der Bundesanstalt für Arbeit.

Enders, Jürgen und Lutz Bornmann. 2002b. Internationale Mobilität bundesdeutscher Promovierter - Eine Sekundäranalyse der Kasseler Promoviertenstudie. Mitteilungen aus der Arbeitsmarkt und Berufsforschung 35: 60-73.

Enders, Jürgen und Alexis-Michel Mugabushaka. 2004. Wissenschaft und Karriere. Erfahrungen und Werdegänge ehemaliger Stipendiaten der DRG. Bonn: Deutsche Forschungsgemeinschaft.

Fassmann, Heinz und Peter Meusburger. 1997. Arbeitsmarktgeographie, Erwerbstätigkeit und Arbeitslosigkeit im räumlichen Kontext. Stuttgart: Teubner.

Faust, Michael. 2002. Karrieremuster von Führungskräften im Wandel. Der Fall Deutschland in vergleichender Perspektive. SOFI-Mitteilungen 30: 69-89.

Featherstone, Mike. 1990. Global Culture. Nationalism. Globalization and Modernity. London: Sage.

Featherstone, Mike, Scott Lash und Roland Robertson. 1995. Global Modernities. London: Sage.

Galinski, Doris. 1986. Brain-Drain aus Entwicklungsländern. Theoretische Grundlagen und entwicklungspolitische Konsequenzen. Frankfurt am Main: Peter Lang.

Giddens, Anthony. 1990. The Consequences of Modernity. Cambridge: Polity Press.

Giddens, Anthony. 1994. Living in a Post-traditional Society. In: Reflexive Modernization. Politicy, Tradition and Aesthetics in the Modern Social Order, hrsg. Ulrich Beck et al., 65-109. Stanford CA: Stanford University Press.

Gieseke, Sunna. 2008. Zur Ärzteakquise nach Wien. Deutsches Ärzteblatt 105/20: A1087-1088, pdf-File auf http://www.aerzteblatt.de/v4/archiv/simplemask.asp, am 13.01.2009.

Glick Schiller, Nina et al. 1992. Towards a transnational perspective on migration, race, class, ethnicity, and nationalism reconsidered. New York: Academy.

Glick Schiller, Nina, L. Basch, and C. Blanc-Szanton. 1995. From Immigrant to Transmigrant:Theorizing Transnational Migration. Anthropology Quarterly 68 (1): 48-63.

Haller, Klaus et al. 2000. Kooperationsmöglichkeiten an der Grenze zwischen Deutschland und Dänemark. Studie über die regionale Wirtschaftsstruktur im Landesteil Schleswig und in Sønderjylland und Suche nach möglichen Kooperationsfeldern sowie künftiger Bedarf des Arbeitsmarktes im grenznahen Raum. Rendsburg: http:/www.uvhord.de/studien/daenemark. pdf am 13.01.2009.

Hartmann, Michael. 2007. Soziale Selektion, Hauskarrieren und geringe Internationalisierung. Personalführung 1: 54-62.

Hillmann, Felicitas und Hedwig Rudolph. 1997. S(Z)eitenwechsel - Internationale Mobilität westlicher Hochqualifizierter am Beispiel Polen. In: Transnationale Migration, Sonderband. Ludger Pries, 245-264. Baden-Baden: Nomos. 
Hunger, Uwe und Dietrich Thränhardt. 2000. Vom Partizipationspostulat zum Civil-Society-Konzept. Zum Perspektivenwechsel in der Integrationsforschung im neuen Jahrhundert. Zeitschrift für Migration und soziale Arbeit 2: 32-39.

IAB; 2007: Migrationseffekte, Zuzug Hochqualifizierter stärkt Innovationskraft der Regionen Aktuelle Analysen aus dem Institut für Arbeitsmarkt- und Berufsforschung der Bundesagentur für Arbeit. IAB Kurzbericht 12/2007.

Internationale Arbeitsmarktbeobachtungsstelle. 2005. Grenzgänger und grenzüberschreitender Arbeitsmarkt in der Großregion. Themenheft im Rahmen des Gesamtvorhabens „Stand, Perspektiven und Handlungserfordernisse des Arbeitsmarkts der Großregion bis 2020“. Saarbrücken.

Jahr, Volker et al. 2002. Internationale Mobilität von Absolventinnen und Absolventen europäischer Hochschulen. Werkstattbericht 61. WZBH Kassel Uni Kassel.

Janssen Manfred. 2001. Grenzüberschreitende Arbeitsmärkte. Realität oder Fiktion? In: Brückenköpfe für die Integration regionaler Arbeitsmärkte?, hrsg. Martin Wenke und Rüdiger Hamm, 115-142. Aachen.

Jerábek, Milan, Jaroslav Dokoupil. 2003. Arbeitsmarkt und Migration im tschechisch-deutschen Grenzraum. In: Die MOE-Staaten vor dem EU Beitritt - Chancen und Probleme aus politikund regionalwissenschaftlicher Sicht. Beiträge zu einem politik- und regionalwissenschaftlichen Symposium an der TU Chemnitz, EZFF Occasional Papers Nr. 27, hrsg. Beate Neuss, Peter Jurczek und Wolfram Hilz. Tübingen http://www.unituebingen. de/ezff/ocp27.html. 13.05. 2008.

Kanter, Moss Rosabeth. 1999. Weltklasse. Im globalen Management lokal triumphieren. Wien: Ueberreuter.

Klemm, Mathias und M. Popp. 2006. Die Lokalität transnationlaer Unternehmen. In: Transnationale Konzerne, Ein neuer Organisationstyp?, hrsg. Ursula Mense-Petermann und Gabriele Wagner, 189-222. Wiesbaden: VS Verlag.

Köhler, Christoph, Kai Loudovici und Olaf Struck. 2007. Generalisierung von Beschäftigungsrisiken oder anhaltende Arbeitsmarktsegmentation?. Berliner Journal für Soziologie 17:387-406.

Kolb, Holger. 2006. Internationale Mobilität von Hochqualifizierten - (k)ein Thema für die Migrationsforschung, In: Neue Zuwanderergruppen in Deutschland, BIB Materialien zur Bevölkerungswissenschaften, hrsg. Frank Swiaczny und Sonja Haug 118: 159-174.

Kommission der Europäischen Gemeinschaften. 2005. Vorschlag für eine Richtlinie des Europäischen Parlamentes und des Rates. Brüssel. http://ec.europa.eu/employment_social/news/ 2005/oct/dir_191005 de.pdf, 14.05.2008.

Kopetsch, Thomas. 2007. Dem deutschen Gesundheitswesen gehen die Ärzte aus! Studie zur Altersstruktur- und Arztzahlentwicklung, 4. Auflg. Bundesärztekammer und Kassenärztliche Bundesvereinigung. Berlin.

Kopetsch, Thomas. 2008. Das Ausland lockt, A716-A719, 105/14 A379-380, pdf-File auf http:// www.aerzteblatt.de/v4/archiv/simplemask.asp, am 13.01.2009.

Kotthoff, Hermann und Alexandra Wagner. 2008. Die Leistungsträger. Führungskräfte im wandel der Firmenkultur - eine Follow-up-Studie. Berlin: edition sigma.

Lucier, Chuck, Steven Wheeler und Rolf Habbel. 2007. The Era of the Inclusive Leader. strategy+business 47.

Luhmann, Niklas. 2000. Organisation und Entscheidung. Opladen/Wiesbaden: Westdeutscher Verlag.

Mense-Petermann, Ursula und Gabriele Wagner. 2006. Transnationale Konzerne, Ein neuer Organisationstyp? Wiesbaden: VS Verlag.

Migrationsbericht des Bundesamtes für Migration und Flüchtlinge im Auftrag der Bundesregierung (Migrationsbericht 2005). 2005. http://www.bundesregierung.de/nn_56708/Content/DE/ Publikation/IB/migrationsbericht-2005.html, am 03.01.2008. 
Münch, Richard. 2001. Offene Räume. Frankfurt/M.: Suhrkamp.

OECD Development Centre. 2007. Policy Coherence for Development: Migration and Developing Countries. Paris: OECD.

Ohmae, Kenichi. 1995. The End of the Nation State. The Rise of Regional Economies. New York et al.: Free Press.

Pohlmann, Markus; H. Gergs. 1997. Manager in Ostdeutschland. Reproduktion oder Zirkulation einer Elite. Kölner Zeitschrift für Soziologie und Sozialpsychologie 49(3): 540-562.

Pohlmann, Markus. 2002. Management, Organisation und Sozialstruktur? Zu neuen Fragestellungen und Konturen der Managementsoziologie. In: Managementsoziologie. Perspektiven, Theorien, Forschungsdesiderate, hrsg. R. Schmidt, H. Gergs und M. Pohlmann, 227-244. Mering: Rainer Hampp Verlag.

Pohlmann, Markus. 2003. Der Generationswechsel und die neue „Weltklasse“ des Managements. Anmerkungen zum Zusammenhang von demographischen und gesellschaftlichen Wandel. ISO-Mitteilungen 2: 50-64.

Pohlmann, Markus. 2009. Globale ökonomische Eliten? Eine Globalisierungsthese auf dem Prüfstand der Empirie. Kölner Zeitschrift für Soziologie und Sozialpsychologie 3 (im Erscheinen)

Pries, Ludger. 1998. Transnationale soziale Räume. In: Perspektiven der Weltgesellschaft, hrsg. Ulrich Beck, 55-88. Frankfurt a. M.: Suhrkamp.

Pries, Ludger. 2003. Gespaltene Migration - gespaltene Gesellschaft? Migranten-Inkorporation in Zeiten der Transnationalisierung. In: Gespaltene Migration, hrsg. Wolfgang Schröer und Stephan Sting, 111-126. Opladen: Leske+Budruch.

Pries, Ludger. 2005a. Migration und transnationale Ökonomie. In: Projekt Migration, hrsg Kathrin Rhomberg und Marion von Osten. Köln: Kölnischer Kunstverein.

Pries, Ludger. 2005b. Arbeitsmigration und Inkorporationsmuster in Europa. In: Zwischen den Welten und amtlichen Zuschreibungen. Neue Formen und Herausforderungen der Arbeitsmigration im 21. Jahrhundert, hrsg. Ders., 15-41. Essen: Klartext.

Pries, Ludger. 2006. Transnational Migration: New Challenges for Nation States and New Opportunities for Regional and Global Development. In: Transnational Migration-Dilemmas, hrsg. Krystyna Iglicka, 9-28. Warszawa: Center for International Relations.

Pries, Ludger. 2008. Die Transnationalisierung der sozialen Welt. Sozialräume jenseits von Nationalgesellschaften. Frankfurt am Main: Suhrkamp.

Richter-Kuhlmann, Eva. 2007. „Ja“ zum Arztberuf, „Nein“ zu deutschen Verhältnissen. Deutsches Ärzteblatt 104/26, A1881-1882, pdf-File auf http://www.aerzteblatt.de/v4/archiv/simplemask.asp, am 13.01.2009.

Richter-Kuhlmann, Eva. 2008. Einbahnstraße ins Ausland. Deutsches Ärzteblatt 105/48, A2547, pdf-File auf http://www.aerzteblatt.de/v4/archiv/simplemask.asp, am 13.01.2009

Robertson, Roland. 1998. Glokalisierung: Homogenität und Heterogenität in Raum und Zeit. In: Perspektiven der Weltgesellschaft, hrsg. Ulrich Beck, 192-221. Frankfurt/M.: Suhrkamp.

Rodriguez-Pose, Andrés. 2003. The European Union, Economy, Society, and Polity. Oxford:University Press.

Rother, Nina. 2005. Migration innerhalb der EU, Wer zieht warum nach Deutschland - und mit welchem Erfolg?. In: Neue Zuwanderergruppen in Deutschland, hrsg. Frank Swiaczny und Sonja Haug, Vorträge der 7. Tagung des Arbeitskreises Migration - Integration - Minderheiten der Deutschen Gesellschaft für Demographie (DGD) in Zusammenarbeit mit dem Soziologischen Institut der Universität Erlangen in Erlangen am 25. November 2005, 41-53. Wiesbaden: Bundesinstitut für Bevölkerungsforschung beim Statistischen Bundesamt.

Salt, John. 1987. Contemporary Trends in International Migration Study. International Migration, 25: 241-250.

Salt, John. 1988. Highly Skilled International Migrants. Careers and Internal Labor Markets. Geoforum 19: 387-399. 
Salt, John. 1989. A Comparative Overview of International Trends and Types 1950-1980. International Migration 23: 431-456.

Sauer, Leonore und Andreas Ette. 2007. Auswanderung aus Deutschland, Stand der Forschung und erste Ergebnisse zur internationalen Migration deutscher Staatsbürger. Wiesbaden: Bundesinstitut für Bevölkerungsforschung beim Statistischen Bundesamt.

Schäuble, Wolfgang. 2007. Konsequenzen der Auswanderung Hochqualifizierter aus Deutschland. Rede von Bundesminister Dr. Wolfgang Schäuble bei der Beratung der Große Anfrage der Abgeordneten Sibylle Laurischk, Rainer Brüderle, Dirk Niebel und weiterer Abgeordneter der FDP-Fraktion am 12. Oktober 2007 im Bundestag, http://www.bmi.bund.de/cln_012/ nn_1136722/Internet/Content/Nachrichten/Reden/2007/10/Rede_BM_Auswanderung _ Hochqualifizierter.html, 13.05.2008.

Schulz, Jonathan. 2005. Grenzgänger in der Euregio Bodensee. Statistik für die Euregio Bodensee. Eine deskriptive Analyse. EURES Bodensee. Amt für Arbeit St. Gallen. translake Konstanz.

Schupp, Jürgen, Janina Söhn und Nicole Schmiade. 2005. Internationale Mobilität von deutschen Staatsbürgern. Chance für Arbeitslose oder Abwanderung der Leistungsträger. Zeitschrift für Bevölkerungswissenschaft 30: 279-292.

Sklair, Leslie. 2001. The Transnational Capitalist Class. Oxford: Blackwell.

Unabhängige Kommission „Zuwanderung“. 2001. Bericht. Zuwanderung gestalten, Integration fördern, pdf-File auf: http://www.bmi.bund.de, am 14.01.2008.

Wagner, Gabriele. 2006. Expatriates als Netzwerkarchitekten. In: Transnationale Konzerne. Ein neuer Organisationstyp?, hrsg. Ursula Mense-Petermann und Gabriele Wagner, 225-248. Wiesbaden: VS Verlag.

Williamson, Oliver E. 1975. Markets and hierarchies: analysis and antitrust implications, a study in the economics of internal organization. New York: Free Press.

\section{Anhang}

1) Forschungsprojekt „Ökonomische Eliten im Wandel““

Tabelle 1: Interviewte nach Position und Grundgesamtheit

\begin{tabular}{lccc}
\hline $\mathrm{N}=89$ & Gesamt & Vorstandsvorsitzende & Vorstände \\
\hline Top 100 Industrie & 49 & 40 & 9 \\
Top 500 Unternehmen & 32 & 26 & 6 \\
ohne Angaben & 8 & 8 & \\
$\quad$ davon: Vorgänger/Nachfolger & 14 & & \\
davon: Unternehmer & 17 & & \\
\hline
\end{tabular}

Tabelle 2: Rücklaufquoten nach Geburtskohorten

\begin{tabular}{lccc}
\hline & $\begin{array}{c}\text { Ältere Generation } \\
(\sim 1930-1940)\end{array}$ & $\begin{array}{c}\text { Mittlere Generation } \\
(1941-1954)\end{array}$ & $\begin{array}{c}\text { Jüngere Generation } \\
(\sim 1955-1965)\end{array}$ \\
\hline Angefragt & 240 & 8 & 25 \\
Geantwortet & $49,6 \%$ & $75,0 \%$ & $92,0 \%$ \\
Interviewt & $27,1 \%$ & $75,0 \%$ & $48,0 \%$ \\
\hline
\end{tabular}


2) Promotionsprojekt: „Managementkonzepte in der Organisation Vergleichende Analysen in Industrieunternehmen und Krankenhäusern“

Tabelle 3: Untersuchungssample

\begin{tabular}{lcc}
\hline & $\begin{array}{c}\text { Interviews, Schriftliche Befragung } \\
\text { Krankenhausmanager }\end{array}$ & $\begin{array}{c}\text { Fallanalysen Unikliniken } \\
\text { Chefärzte und Ärztliche Direktoren }\end{array}$ \\
\hline Bisher realisiert & 28 & 94 \\
\hline
\end{tabular}

Markus Pohlmann ist Professor für Soziologie an der Universität Heidelberg. Forschungsbereich: Industrie-, wirtschafts- und organisationssoziologische Studien, Forschungsschwerpunkte: Wandlungsdynamiken des Kapitalismus im internationalen Vergleich; Organisation und Management. Veröffentlichungen: Globale ökonomische Eliten - Eine Globalisierungsthese auf dem Prüfstand der Empirie (Kölner Zeitschrift für Soziologie und Sozialpsychologie 2009, im Erscheinen); Die neue Kulturtheorie und der Geist des Kapitalismus - Max Weber and beyond, in: Hessinger, Philipp et al. (Hg.): Der neue Geist des Kapitalismus? (VS 2008); Der diskrete Charme der Bourgeoisie? Zur Soziologie des modernen Wirtschaftsbürgertums, in: Sigmund, Steffen et al. (Hg.): Soziale Konstellationen und historische Analysen (Mohr Siebeck 2008). Adresse: Ruprecht-Karls-Universität Heidelberg, Institut für Soziologie, Bergheimer Straße 58, D-69115 Heidelberg.

Stefan Bär, Doktorand, wissenschaftlicher Mitarbeiter am Institut für Soziologie, Universität Heidelberg. Forschungsschwerpunkt: Krankenhausmanagement. Veröffentlichungen: Wege ins Krankenhaus, Krankenhauswahl als Rational Choice. (VDM 2008); Die Qual der Wahl - oder ist doch alles ganz einfach? Anmerkungen zur Erklärung von Krankenhauswahl-Entscheidungen. Das Krankenhaus (2008); Patientenrekrutierung oder bewusste Auswahl? Arzt und Krankenhaus (2009). Adresse: Ruprecht-Karls-Universität Heidelberg, Institut für Soziologie, Bergheimer Straße 58, D-69115 Heidelberg. 